Manuscript Number:

Title: Pore-Forming Bioinks to Enable Spatio-Temporally Defined Gene Delivery in Bioprinted Tissues

Article Type: Research paper

Keywords: Non-viral gene delivery, bioink, tissue engineering, 3D printing, gene activated matrix, osteochondral regeneration

Corresponding Author: Dr. Daniel J. Kelly,

Corresponding Author's Institution: Trinity College Dublin

First Author: Tomas Gonzalez Fernandez, PhD

Order of Authors: Tomas Gonzalez Fernandez, PhD; Swetha Rathan, PhD; Christopher Hobbs; Pierluca Pitacco; Fiona Freeman, PhD; Grainne M Cunniffe, PhD; Nicholas J Dunne, PhD; Helen O McCarthy, PhD; Valeria Nicolosi, PhD; Fergal J O'Brien, PhD; Daniel J. Kelly

Abstract: The regeneration of complex tissues and organs remains a major clinical challenge. With a view towards bioprinting such tissues, we developed a new class of pore-forming bioink to spatially and temporally control the presentation of therapeutic genes within bioprinted tissues. By blending sacrificial and stable hydrogels, we were able to produce bioinks whose porosity increased with time following printing. When combined with amphipathic peptide-based plasmid DNA delivery, these bioinks supported enhanced non-viral gene transfer to stem cells in vitro. By modulating the porosity of these bioinks, it was possible to direct either rapid and transient (pore-forming bioinks), or slower and more sustained (solid bioinks) transfection of host or transplanted cells in vivo. To demonstrate the utility of these bioinks for the bioprinting of spatially complex tissues, they were next used to zonally position stem cells and plasmids encoding for either osteogenic (BMP2) or chondrogenic (combination of TGF- 33 , BMP2 and SOX9) genes within networks of 3D printed thermoplastic fibers to produce mechanically reinforced, gene activated constructs. In vivo, these bioprinted tissues supported the development of a vascularised, bony tissue overlaid by a layer of stable cartilage. When combined with multiple-tool biofabrication strategies, these gene activated bioinks can enable the bioprinting of a wide range of spatially complex tissues.

Suggested Reviewers: Magali Cucchiarini PhD Professor in Molecular Biology, Center of Experimental Orthopaedics, Saarland University Medical Center mmcucchiarini@hotmail.com Expert in the field of gene delivery for orthopedic tissue engineering

Farshid Guilak PhD

Professor, Department of Orthopaedics, Washington University guilak@wustl. edu 
Expert in controlled release and gene therapy for musculoskeletal tissue engineering

Jason A Burdick PhD

Professor, Department of Bioengineering, University of Pennsylvania burdick2@seas.upenn.edu

Expert in tissue engineering and biofabrication for musculoskeletal regeneration

Jacqueline Alblas PhD

Associate Professor, Department of Orthopaedics, UMC Utrecht

j.alblas@umcutrecht.nl

Expert in non-viral gene delivery for tissue engineering applications 
Paper Title: Pore-Forming Bioinks to Enable Spatio-Temporally Defined Gene Delivery in Bioprinted Tissues

Authors: Tomas Gonzalez-Fernandez, Swetha Rathan, Christopher Hobbs, Pierluca Pitacco, Fiona Freeman, Grainne M. Cunniffe, Nicholas J. Dunne, Helen O. McCarthy, Valeria Nicolosi, Fergal J. O'Brien, Daniel J. Kelly

Dear editor,

We hereby submit the manuscript entitled "Pore-Forming Bioinks to Enable SpatioTemporally Defined Gene Delivery in Bioprinted Tissues". We declare that this manuscript is original, has not been published before and is not currently being considered for publication elsewhere.

Statement of significance:

Successful 3D bioprinting of complex tissues and organs will require the development of new bioinks that can spatially and temporally control the presentation of regulatory biomolecules to cells. To this end, we engineered a new class of bioink whose porosity increases post-printing to modulate cellular uptake of vector-plasmid complexes resulting in either rapid and transient, or slower and more sustained transfection of host or transplanted cells in vitro and in vivo. As a proof of concept for the application of these gene activated bioinks to spatially direct stem cell differentiation, we positioned appropriate gene/non-viral vector/bioink combinations in bioprinted implants. In vivo, these bioprinted tissues supported the development of a vascularised, bony tissue overlaid by a layer of stable cartilage resembling the structure of a typical musculoskeletal interface.

This study demonstrates for the first time that gene delivery and biofabrication techniques can be combined to allow the engineering of spatially complex tissues. We therefore believe that this interdisciplinary study will be of great interest to the gene delivery, controlled release, biofabrication, and regenerative medicine communities.

Sincerely,

Prof. Daniel Kelly

Director, Trinity Centre for Bioengineering

E-mail: kellyd9@tcd.ie 
Graphical Abstract

\section{Development of pore-forming bioinks}

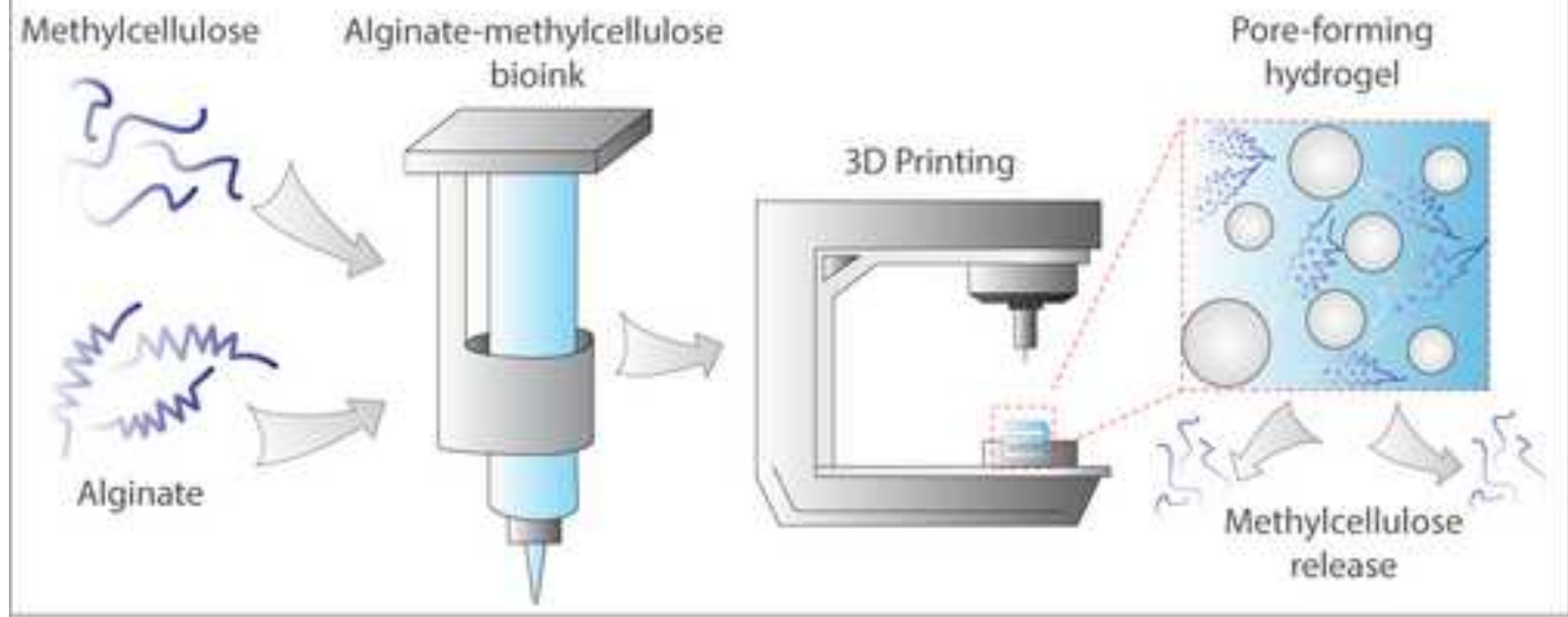

\section{Pore-forming bioinks enhance nanoparticle-mediated gene delivery}

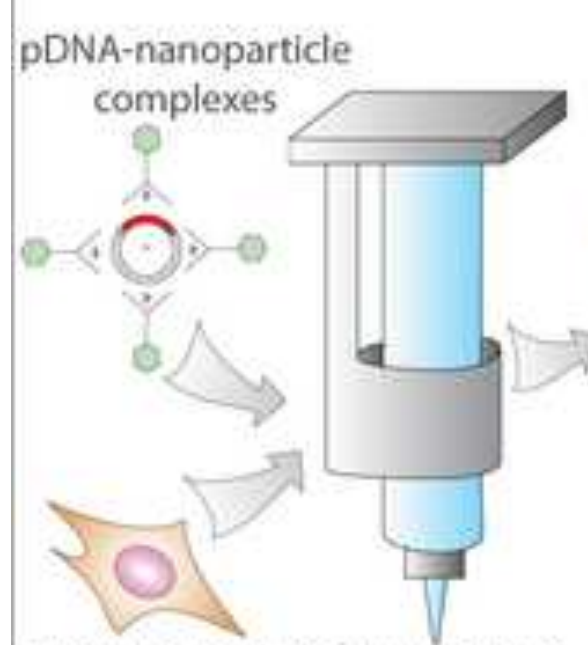

Mesenchymal Gene-activated stem cell bioink
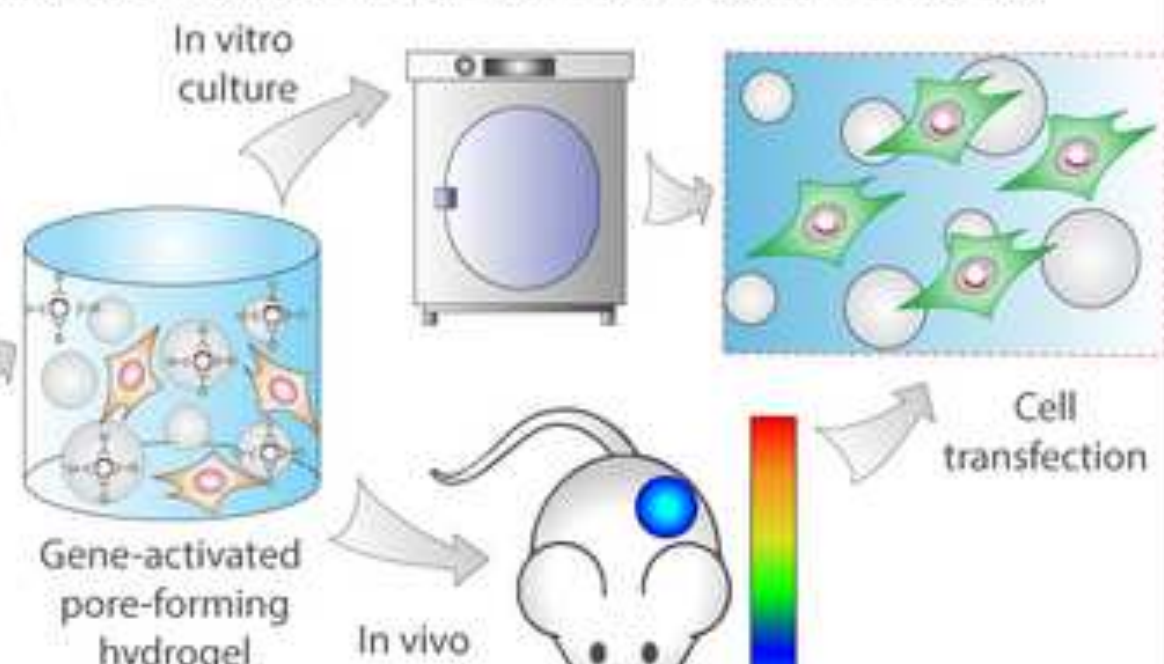

Gene-activated pore-forming hydrogel

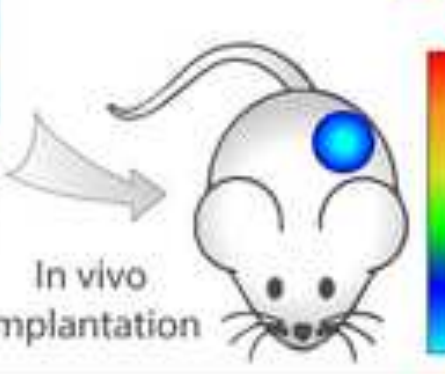

Cell transfection

\section{Therapeutic gene delivery to spatially direct stem cell fate}

\section{Gene-activated}

pore-forming bioink

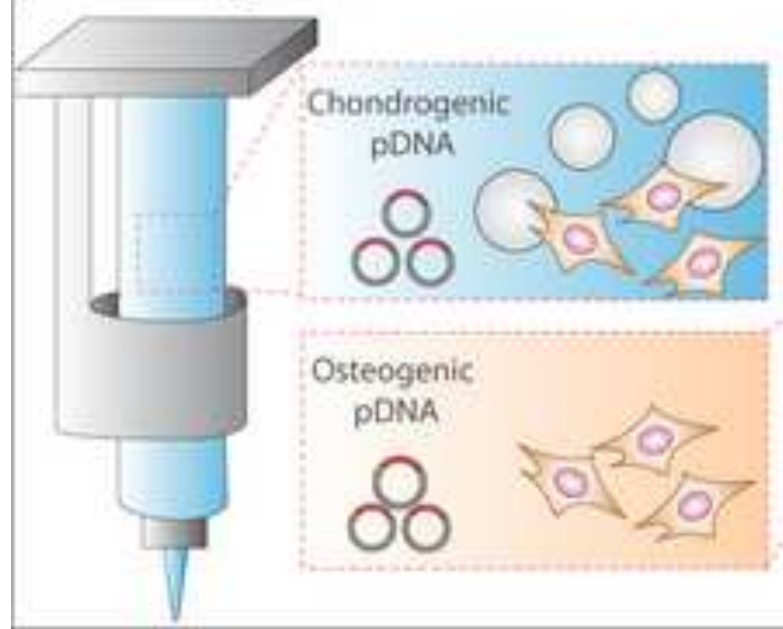

Gene-activated solid bioink

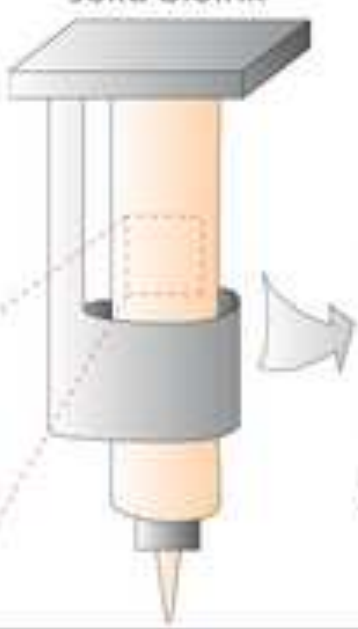

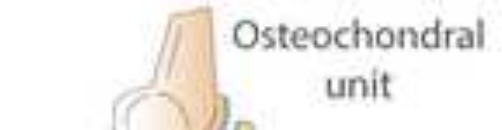

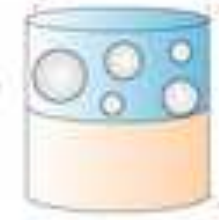

Multilayered constructs
Chondrogenesis

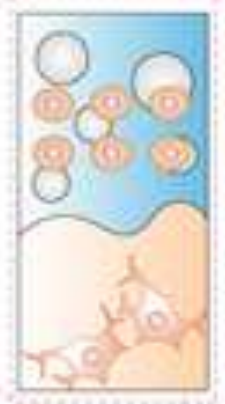

Osteogenesis 


\section{Pore-Forming Bioinks to Enable Spatio-Temporally Defined Gene Delivery in Bioprinted Tissues}

Gonzalez-Fernandez T. ${ }^{1,2,3}$, Rathan S. ${ }^{1,2}$, Hobbs C. ${ }^{3,4,5}$, Pitacco P. ${ }^{1,2}$, Freeman F.E. ${ }^{1,2}$, Cunniffe

G.M. ${ }^{1,2}$, Dunne N.J. ${ }^{1,2,6,7}$, McCarthy H.O. ${ }^{7}$, Nicolosi V. ${ }^{3,4,5}$, O'Brien F.J. ${ }^{1,3,9}$, Kelly D.J. ${ }^{1,2,3,9^{*}}$

${ }^{1}$ Trinity Centre for Bioengineering, Trinity Biomedical Sciences Institute, Trinity College Dublin.

${ }^{2}$ Department of Mechanical and Manufacturing Engineering, School of Engineering, Trinity College Dublin.

${ }^{3}$ Advanced Materials and Bioengineering Research Centre, Trinity College Dublin and Royal College of Surgeons in Ireland.

${ }^{4}$ School of Physics, Trinity College Dublin, Ireland.

${ }^{5}$ Centre for Research of Adaptive Nanostructures and Nanodevices, Trinity College Dublin, Ireland.

${ }^{6}$ Centre for Medical Engineering Research, School of Mechanical and Manufacturing Engineering, Dublin City University, Ireland.

${ }^{7}$ School of Mechanical and Manufacturing Engineering, Dublin City University, Ireland.

${ }^{8}$ School of Pharmacy, Queen's University Belfast, Northern Ireland.

${ }^{9}$ Tissue Engineering Research Group, Dept. of Anatomy, Royal College of Surgeons in Ireland.

*Corresponding author: Daniel J Kelly

e-mail: kellyd9@tcd.ie

Trinity Centre for Bioengineering,

152-160 Pearse street,

Dublin 2, Ireland

Phone: +353-1-8963947

Fax: +353-1-6795554

Key words: Non-viral gene delivery, bioink, tissue engineering, 3D printing, gene activated matrix, osteochondral regeneration 


\begin{abstract}
The regeneration of complex tissues and organs remains a major clinical challenge. With a view towards bioprinting such tissues, we developed a new class of pore-forming bioink to spatially and temporally control the presentation of therapeutic genes within bioprinted tissues. By blending sacrificial and stable hydrogels, we were able to produce bioinks whose porosity increased with time following printing. When combined with amphipathic peptide-based plasmid DNA delivery, these bioinks supported enhanced non-viral gene transfer to stem cells in vitro. By modulating the porosity of these bioinks, it was possible to direct either rapid and transient (pore-forming bioinks), or slower and more sustained (solid bioinks) transfection of host or transplanted cells in vivo. To demonstrate the utility of these bioinks for the bioprinting of spatially complex tissues, they were next used to zonally position stem cells and plasmids encoding for either osteogenic (BMP2) or chondrogenic (combination of TGF- $\beta 3$, BMP2 and SOX9) genes within networks of 3D printed thermoplastic fibers to produce mechanically reinforced, gene activated constructs. In vivo, these bioprinted tissues supported the development of a vascularised, bony tissue overlaid by a layer of stable cartilage. When combined with multiple-tool biofabrication strategies, these gene activated bioinks can enable the bioprinting of a wide range of spatially complex tissues.
\end{abstract}




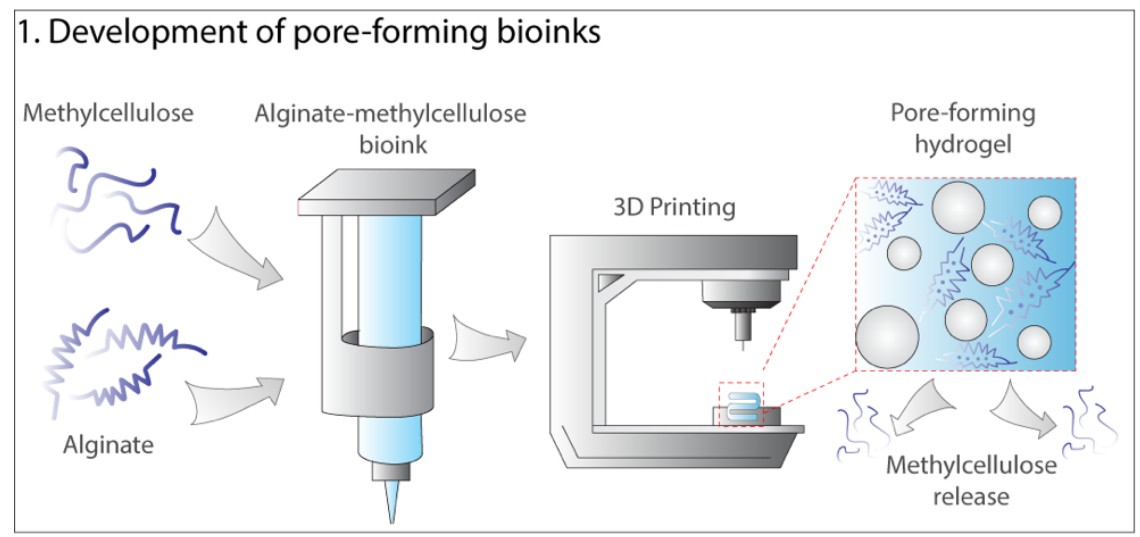

2. Pore-forming bioinks enhance nanoparticle-mediated gene delivery

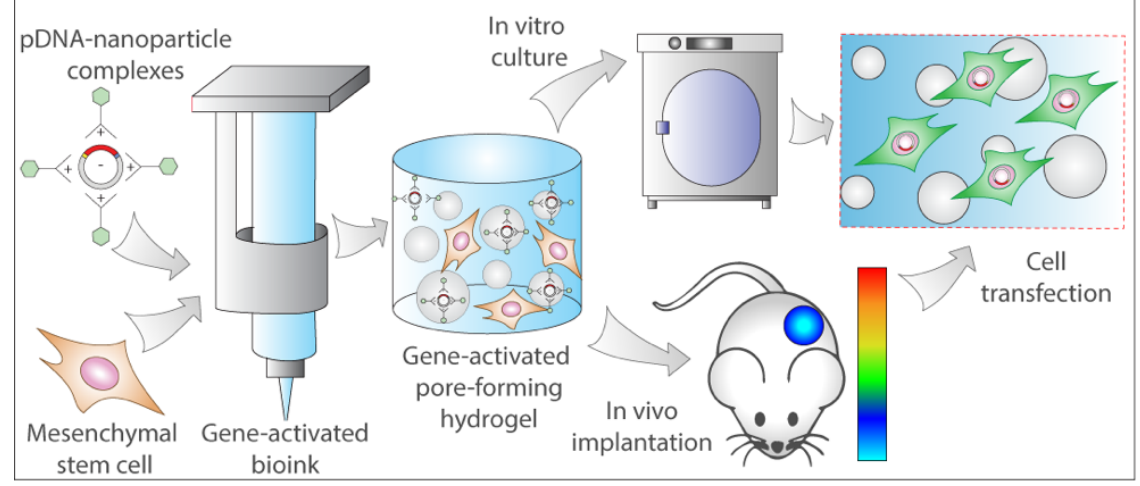

3. Therapeutic gene delivery to spatially direct stem cell fate

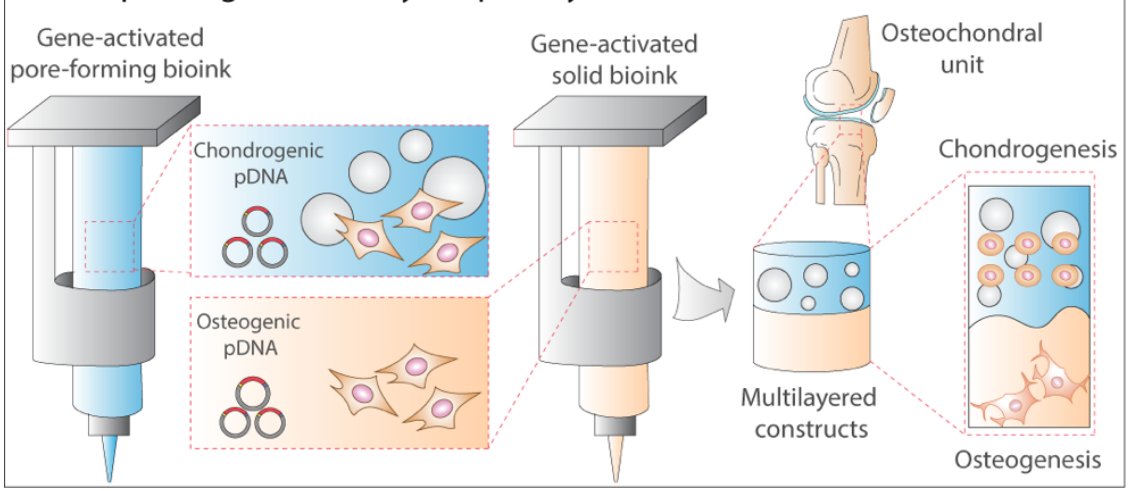

Graphical abstract 


\section{Introduction}

Tissue engineering has enabled the regeneration of relatively simple and homogenous tissues, but the engineering of more complex interfaces and solid organs remains challenging. This has led to increased interest in the field of bioprinting, which typically involves the layerby-layer deposition of different cell containing bioinks and supporting biomaterials, to engineer constructs that mimic the biological and/or mechanical function of specific tissues[1-5]. While this approach can enable the bioprinting of spatially complex tissues through depositing multiple different cell types within a single construct, it is limited by the availability of functional primary human cells and the associated complexity with the use of such cell sources in a clinical setting[6]. An alternative approach would be printing a single population of undifferentiated stem cells[7-9], or to recruit such a cell population following implantation[10,11], where the printed construct provides appropriate instructions to direct stem cell differentiation and tissue organisation in vivo. For such an approach to be successful, it requires the precise presentation of regulatory cues within the printed construct to direct cells to form appropriate tissue types in the correct anatomical location. The precise temporal and spatial presentation of signaling molecules such as growth factors can be challenging as they can rapidly diffuse through implanted hydrogels or scaffolds[12]. Additionally, such delivery systems typically possess burst release kinetics[13], limiting drug availability at the site of injury and its therapeutic effect. In order to overcome these limitations, delivering high concentrations of growth factors is often required to elicit a therapeutic effect, which can result in cytotoxicity and undesired sideeffects[14,15].

Engineering cells to locally produce growth and transcription factors through gene delivery represents an alternative approach to precisely control stem cell differentiation within bioprinted tissues[12]. Such genetic modification allows for cell-mediated production of proteins with authentic post-translational modifications and increased biological activity in comparison to their recombinant counterparts[16]. The incorporation of the gene of interest and its delivery vector into a hydrogel, to form a gene activated bioink (GAB), offers a promising platform for localized, sustained and spatially defined delivery of therapeutics to transplanted and/or host cells for the repair of tissues and organs in situ.

Here, we developed a new class of non-viral gene activated bioink that enables both temporal and spatial control of plasmid gene delivery to stem cells for the bioprinting of complex tissues. To temporally modulate mesenchymal stem cell (MSC) gene transfection within printed tissues, we engineered bioinks whose porosity increases post-printing to modulate cellular 
uptake of vector-plasmid complexes. In vivo these pore-forming bioinks accelerated the transfection of either host cells or implanted cells. To demonstrate the utility of these GABs, they were next used to direct the differentiation of mesenchymal stem cells (MSCs) along different pathways both in vitro and in vivo. We show that by spatially positioning appropriate gene/non-viral vector/bioink combinations in mechanically reinforced bioprinted tissues, it is possible to engineer a bone-soft tissue interface in vivo, consisting of a cartilaginous matrix overlaying a vascularized bone tissue, resembling the architecture of the osteochondral unit. These findings highlight the potential of pore-forming bioinks delivering therapeutic genes to spatially direct stem cell differentiation and thereby enable the engineering of complex tissues.

\section{Materials and Methods}

\subsection{Alginate-methylcellulose hydrogel preparation and characterization}

Methylcellulose (Sigma-Aldrich, Ireland), with a molecular weight of $40,000 \mathrm{Mn}$ and a viscosity of $400,000 \mathrm{Cp}$, was added in a ratio of $1: 1$ or $1: 2$ to a solution of $2 \%$ alginate (Pronova FMC BioPolymer, Norway). The alginate only and ALG-MC hydrogels were crosslinked using a 3\% agarose/100 $\mathrm{nM} \mathrm{CaCl}_{2}$ (both from Sigma-Aldrich, Ireland) mold to form cylindrical constructs ( $\varnothing 5$ x H4 mm). After fabrication and incubation of the gels at room temperature (RT) in ultrapure water (UPW), their macroscopic and microscopic structure was analysed. For histological analysis, constructs were processed as previously described [17] and stained with hematoxylin and eosin (H\&E) (Sigma-Aldrich, Ireland). The microstructure of hydrogel-based constructs was characterized using cryogenic SEM (cryOSEM) methods. Samples were prepared for imaging by mounting onto standard SEM stubs, then subsequently plunged into liquid nitrogen slush, freeze fractured and lightly sputter coated before transferring to the SEM chamber (Quorum Technologies, UK). Samples were imaged at 5 kV using a Carl Zeiss Ultra SEM (Carl Zeiss, Germany). Pore size was analysed using ImageJ analysis and calculated using Feret's diameter [18]. The percentage of wet weight $(w / w)$ loss was calculated by weighting the constructs immediately after fabrication and after 7 days of incubation in UPW at RT.

\subsection{Mechanical testing}

Constructs were mechanically tested $(n=4)$ in unconfined compression using a standard material testing machine with a $5 \mathrm{~N}$ load cell (Zwick Roell Z005). Briefly, constructs were kept hydrated through immersion in DMEM (Gibco Biosciences, Ireland) bath maintained at RT. A preload of $0.01 \mathrm{~N}$ was applied to ensure that the construct surface was in direct contact with the impermeable loading platens. Stress relaxation tests were performed consisting of a ramp displacement of $1 \mathrm{~m} / \mathrm{s}$ up to $10 \%$ strain. A relaxation period of $30 \mathrm{~min}$ was used. 


\subsection{Rheological assessment}

MCR 301 rheometer (Anton Paar GmbH, Austria) was used for the rheological assessment of the hydrogels. A $50 \mathrm{~mm}$ parallel plate with a measurement gap of $0.55 \mathrm{~mm}$ was used. Shear rates in the range of 0.1 to $1000 \mathrm{1} / \mathrm{s}$ at the frequency of $1 \mathrm{~Hz}$ were used in order to determine the linear viscoelastic range of the samples. A recovery test was also carried out where the hydrogels were subjected to 1 1/s shear rate for $60 \mathrm{~s}, 100 \mathrm{1} / \mathrm{s}$ shear rate for $10 \mathrm{~s}$ and $11 / \mathrm{s}$ shear rate for $60 \mathrm{~s}$ to simulate the printing process, and to assess the recovery of the viscosity of the materials[19]. The 100 1/s shear rate is estimated to be the maximum shear rate experienced by the hydrogel during printing process. This test also informs the thixotropic property of the hydrogels which is how quickly the hydrogel can recover its viscosity immediately after printing[19].

\subsubsection{D printing and printability assessment}

A 3D Discovery multi-head (Regen HU, Switzerland) 3D plotter was used for printing. For hydrogel printing, pneumatic driven syringes with 25 Gauge (G) needles were used at extrusion pressures between 0.1 and $0.2 \mathrm{MPa}$. The filament spreading ratio, defined as the width of the printed filament divided by the needle diameter, was used as a measure of printability as previously described [20]. For polycaprolactone (PCL) (Mw = 45,000 Mn, Sigma-Aldrich, Ireland) printing, fuse deposition modeler (FDM) was used to deposit filaments of molten PCL. To characterize the filament size and separation between fibers, PCL scaffolds were placed onto SEM stubs and $\mathrm{Au} / \mathrm{Pd}$ sputter coated before imaging. SEM characterization was conducted on a Carl Zeiss Supra SEM, aligned at 5 kV (Carl Zeiss, Germany).

\subsection{Isolation and expansion of bone marrow-derived MSCs, and chondrogenic differentiation}

Bone marrow-derived MSCs were isolated from the femora of porcine donors (3-4 months, $>50$ $\mathrm{kg}$ ) within $3 \mathrm{~h}$ of sacrifice according to a modified method developed for human MSCs [21]. Mononuclear cells were plated at a seeding density of $5 \times 10^{3}$ cells $/ \mathrm{cm}^{2}$ in standard culture media, high glucose Dulbecco's modified Eagle's medium $(4.5 \mathrm{mg} / \mathrm{mL}$ D-glucose and $200 \mathrm{mM} \mathrm{L-}$ glutamine; hgDMEM) supplemented with $10 \%$ fetal bovine serum (FBS) and penicillinstreptomycin (100 U/mL) (all from Gibco, Biosciences, Ireland), and expanded in a humidified atmosphere at $37{ }^{\circ} \mathrm{C}, 5 \% \mathrm{CO}_{2}$, and $20 \% \mathrm{pO}_{2}$. MSCs at passage 2-3 were used for all experiments. For chondrogenic differentiation of MSCs, MSCs were trypsinized and encapsulated at a concentration of $20 \times 10^{6}$ cells $/ \mathrm{mL}$, in either pre-crosslinked alginate hydrogels or ALG-MC hybrid gels. The cell laden bioinks were printed to form cylindrical constructs that were further crosslinked in a $100 \mathrm{mM} \mathrm{CaCl}_{2}$ bath for 5-10 min. After crosslinking the gels were cultured for 
four weeks at $5 \% \mathrm{PO}_{2}$ in chondrogenically defined media consisting of DMEM GlutaMAX supplemented with $100 \mathrm{U} / \mathrm{mL}$ penicillin/streptomycin (both Gibco), $100 \mu \mathrm{g} / \mathrm{mL}$ sodium pyruvate, $40 \mu \mathrm{g} / \mathrm{mL}$ L-proline, $50 \mu \mathrm{g} / \mathrm{mL}$ L-ascorbic acid 2-phosphate, $4.7 \mu \mathrm{g} / \mathrm{mL}$ linoleic acid, 1.5 $\mathrm{mg} / \mathrm{mL}$ bovine serum albumin, $1 \mathrm{x}$ insulin-transferrin-selenium, $100 \mathrm{nM}$ dexamethasone (all from Sigma-Aldrich) and $10 \mathrm{ng} / \mathrm{mL}$ human TGF- $\beta 3$ (PrepoTech, UK).

\subsection{Plasmid propagation}

Six different plasmids were used in the current study: three plasmids encoding for the reporter genes green fluorescent protein (pGFP, Amaxa, Lonza Cologne AG, Germany) and luciferase (pLUC, pGaussia Luciferase; New England Biolabs, Massachusetts, USA), and dtTomato (pTomato, kind donation from Prof. Gerhart Ryffel through Addgene), and another three encoding for the therapeutic genes BMP2 (pBMP2, kind donation from Prof. Kazihusa Bessho, Kyoto University, Japan), TGF- $\beta 3$ (pTGF- $\beta 3$, InvivoGen, Ireland) and SOX9 (pSOX9, kind donation from Prof. Dr. Gun-II Im, Dongguk University, South Korea). Plasmid amplification was performed by transforming chemically competent Escherichia coli bacterial cells (One Shot TOP10; Biosciences, Ireland) according to the manufacturer's protocol. The transformed bacteria were cultured on LB plates with $50 \mathrm{mg} / \mathrm{L}$ kanamycin (Sigma-Aldrich, Ireland) as the selective antibiotic for pGFP and pSOX9, and $100 \mathrm{mg} / \mathrm{L}$ ampicillin (Sigma-Aldrich, ireland) as the selective antibiotic for pLUC, pTGF- $\beta 3$, pBMP2 and pTomato. Bacterial colonies were harvested and inoculated in LB broth (Sigma-Aldrich, Ireland) and incubated overnight for further amplification. The harvested bacterial cells were then lysed, and the respective pDNA samples were purified using qiagen plasmid kit (MaxiPrep Kit; Qiagen, Ireland). Nucleic acid concentration ( $\mathrm{ng} / \mathrm{mL}$ ) was determined by analyzing the 260:280 ratio and $230 \mathrm{~nm}$ measurement using NanoDrop spectrophotometer (Labtech International, UK).

\subsection{Preparation of delivery vectors, vector-pDNA complexes}

The synthesis of the nHA particles was performed as previously described[22]. nHA-pDNA complexes were prepared by adding $150 \mathrm{~mL}$ of the $\mathrm{nHA}$ solution to $2 \mathrm{mg}$ of pDNA pretreated with $0.25 \mathrm{M} \mathrm{CaCl}_{2}$ (Sigma-Aldrich, Ireland) as previously optimized[23,24]. The RALA peptide was produced as previously described[25], and complexed with pDNA at an n:p ratio of 10 for $30 \mathrm{~min}$ at RT.

\subsection{Monolayer transfection and 3D printing of pre-transfected cells}

For monolayer transfection, MSCs were seeded in T175 flasks at a seeding density of $5 \times 10^{4}$ cells $/ \mathrm{cm}^{2}$ and cultured for $72 \mathrm{~h}$ in standard culture media prior to transfection. RALA and nHA- 
pDNA complexes were prepared immediately before transfection. For the RALA-pDNA transfections, the plated cells were washed twice with PBS and incubated in $10 \mathrm{~mL}$ of Opti-MEM (Life Technologies, Ireland) for $2 \mathrm{~h}$. After the incubation time, complexes were suspended in 5 $\mathrm{mL}$ of Opti-MEM and added to the MSCs to a density of $0.2 \mu \mathrm{g}$ of pDNA $/ \mathrm{cm}^{2}$. For nHA-pDNA transfection, complexes were suspended in $5 \mathrm{~mL}$ of standard media and added to the MSCs at the same density as the RALA-pDNA complexes. Following incubation for 4-6 $\mathrm{h}$ with both RALA and nHA-pDNA complexes, the cells were washed with PBS and standard culture media was added. 3 days after transfection, cells were trypsinized and encapsulated in pre-crosslinked alginate or ALG-MC paste to a final concentration of $20 \times 10^{6}$ cells $/ \mathrm{mL}$. Once encapsulated, cell laden gels were printed simultaneously using different pneumatic heads of the 3D Discovery system.

\subsection{Preparation of gene activated bioinks and in vitro culture of gene activated constructs}

For $1 \mathrm{~mL}$ of pre-crosslinked $\mathrm{CaCl}_{2}$ and ALG-Mc bioinks, $20 \mu \mathrm{g}$ of pDNA were complexed to either $\mathrm{nHA}(0.0125 \mu \mathrm{L} \mathrm{nHA} / \mu \mathrm{g}$ pDNA) or RALA (N:P ratio of 10$)$ and added to a suspension of MSCs (20 $\times 10^{6}$ cells $/ \mathrm{mL}$ bioink) in either $0.5 \mathrm{~mL}$ of standard media for $\mathrm{nHA}$ or $0.5 \mathrm{~mL}$ of Opti-MEM for RALA transfection. After $1 \mathrm{~h}$ of incubation, the nHA-pDNA containing groups were spun down to retire excess of $\mathrm{CaCl}_{2}$ and mixed with $\mathrm{CaCl}_{2}(50 \mathrm{mM})$ and $3.5 \%$ alginate added in a 7:3 ratio until a homogenous mixture of a final $2 \%$ alginate was obtained. For the RALA-pDNA containing groups, the $0.5 \mathrm{~mL}$ of cell and complex suspension were mixed with $0.5 \mathrm{~mL}$ of $4 \%$ ALG-MC paste 1 : 2 ratio) until a homogenous mixture of a final $2 \%$ alginate was obtained. After $3 D$ printing and crosslinking of the constructs in a $100 \mathrm{mM} \mathrm{CaCl}_{2}$ bath for 5-10 min, RALA-pDNA containing 3D printed hydrogels were incubated for 4-6 hours in Opti-MEM. In contrast, after crosslinking, nHA-pDNA containing gels were directly cultured in the desired media. For reporter gene expression, constructs were incubated at $37{ }^{\circ} \mathrm{C}$ and $20 \% \mathrm{PO}_{2}$ in standard culture media. For therapeutic gene delivery, constructs were incubated at $37^{\circ} \mathrm{C}$ and $5 \% \mathrm{PO}_{2}$ in chemically defined chondrogenic media without recombinant TGF- $\beta 3$ supplementation.

\subsection{Characterisation of RALA-pDNA encapsulation and release in ALG-MC hydrogels}

RALA-pDNA complexes ( $N$ :P ratio of 10 ) were formed and encapsulated in alginate and ALG-MC cells at a concentration of $0.5 \mu \mathrm{g} / \mathrm{mL}$. To assess the complex encapsulation efficiency, after crosslink in a $3 \%$ agarose/100 $\mathrm{nM} \mathrm{CaCl} 2$ mold to form cylindrical constructs, they were homogenized in citrate buffer for $1 \mathrm{~h}$ at $37^{\circ} \mathrm{C}$ and the amount of pDNA per gel was quantified with a nanodrop. To characterize complex release, after crosslinking, gels were incubated in 
UPW at RT. The UPW was collected and changed after 12 h, 24 h, 3 days, 7 days and 10 days, and the quantity of pDNA in the UPW was analysed with a nanodrop.

\subsection{Reporter and therapeutic gene expression analysis}

For gels containing pGFP, transfected cells were assessed using a Leica SP8 scanning confocal microscope (Leica Microsystems, Ireland). Luciferase expression was assessed in the culture media using a Pierce Gaussia Luciferase Flash Assay Kit (ThermoFisher, Ireland) at different time points up to 10 or 14 days. The levels of BMP2 and TGF- $\beta 3$ were quantified in the culture medium using ELISAs (R\&D Systems) according to the manufacturer's instructions.

\subsection{Live dead analysis}

Cell viability was assessed using a LIVE/DEAD viability/cytotoxicity assay kit (Invitrogen, Bioscience, Ireland). Briefly, constructs were cut in half, washed in phosphate-buffered saline (PBS) followed by incubation for $1 \mathrm{~h}$ in PBS containing $2 \mathrm{mM}$ calcein and $4 \mathrm{mM}$ ethidium homodimer1. Sections were again washed in PBS, imaged using a Leica SP8 scanning confocal microscope.

\subsection{Subcutaneous in vivo construct implantation and in vivo luciferase analysis}

Control and gene activated 3D printed constructs were implanted subcutaneously into the back of nude mice (Balb/c; Harlan, United Kingdom) as previously described with two samples from the same group inserted in each of two pockets [26]. The constructs were harvested after three weeks for the in vivo luciferase expression study, and after four weeks for the therapeutic genes studies. Mice were euthanized by $\mathrm{CO}_{2}$ inhalation and the animal protocol was reviewed and approved by the Ethics Committee of Trinity College Dublin and the Health Products Regulatory Authority (HPRA). For the in vivo luciferase imaging, a protocol previously developed by Tannous et al (2009) was followed [27]. Briefly, previous to administration, coelenterazine (Gold Biotechnology, USA), prepared in acid methanol at a concentration of $5 \mathrm{mg} / \mathrm{mL}$, was dissolved in PBS to a final concentration of $0.5 \mathrm{mg} / \mathrm{mL}$ under minimal light conditions, immediate after the mice were anesthetized using isoflurane, $50 \mu \mathrm{L}$ of the coelenterazine mix were injected in each of the pockets. Immediately after coelenterazine injection, photon counts were acquired using a real-time bioluminescence imaging system (Photonlmager; Biospace lab, France) over five minutes.

\subsection{Micro-computed tomography analysis}

Micro-computed tomography (microCT) scans were performed using a Scanco Medical 40 microCT system (Scanco Medical, Switzerland) with a $70 \mathrm{kVp}$ X-ray source at $114 \mu \mathrm{A}$. 
Quantification was performed by setting a threshold of 200 , corresponding to a density of $411.98 \mathrm{mg}$ hydroxyapatite $/ \mathrm{cm}^{3}$ and the mineral volume $\left(\mathrm{mm}^{3}\right)$ was recorded. Reconstructed 3D images were generated from the scans and used to visualize mineral distribution throughout the constructs.

\subsection{Bilayer construct fabrication}

Control or gene activated hydrogels were casted into 3D printed PCL constructs ( $\varnothing 6 \times \mathrm{H} 5 \mathrm{~mm}$ ) inserted in $3 \%$ agarose $/ 100 \mathrm{nM} \mathrm{CaCl} 2$ molds, to form cylindrical constructs ( $\varnothing 6 \times \mathrm{H} 6 \mathrm{~mm}$ ) comprising of an osteogenic layer ( $\mathrm{H} 4 \mathrm{~mm})$ and a chondrogenic layer $(\mathrm{H} 2 \mathrm{~mm})$. The osteogenic layer was formed by either MSC laden pre-crosslinked alginate gel for the control groups or MSC laden pre-crosslinked alginate gel containing nHA-pBMP2 complexes for the gene activated groups. The chondrogenic layer was formed by either MSC laden ALG-MC (1:2 ratio) hybrid gels for the control groups, or MSC laden ALG-MC hybrid gels containing either RALA-pTGF- $\beta 3$ pBMP2 (pTGF-pBMP) or RALA-pTGF- $33-p B M P 2-p S O X 9$ (pTGF-pBMP-pSOX) gene combinations.

\subsection{Biochemical analysis}

DNA and sGAG content were quantified biochemically using the Hoechst Bisbenzimide 33258 dye assay and the dimethyl methylene blue dye-binding (DMMB) assay respectively, as previously described [28]. To exclude any background absorbance from the alginate, the $\mathrm{pH}$ of the DMMB was adjusted to 1.35 . Total collagen content was determined by measuring the hydroxyproline content using the dimethylaminobenzaldehyde and chloramine $\mathrm{T}$ assay and a hydroxyproline to collagen ratio of 1:7.69. Calcium content was determined using a Sentinel Calcium Kit (Alpha Laboratories Ltd, UK) after digestion in $1 \mathrm{M} \mathrm{HCl}$ at $110{ }^{\circ} \mathrm{C}$ for $48 \mathrm{~h}$.

\subsection{Histological and Immunohistochemical Analysis}

Constructs were processed for histological analysis as previously described [17]. The sections were stained with H\&E to assess bone and blood vessel formation, aldehyde fuschin/alcian blue to assess SGAG content, picrosirius red to assess collagen content and alizarin red to assess calcification. Collagen types I, II, and X were evaluated using a standard immunohistochemical technique as previously described [29]. The presence of vascular structures was quantified by counting distinct areas of red blood cell activity as a blood vessel. The number of blood vessels across a whole cross section was then counted.

\subsection{Statistical analysis}


Statistical analyses were performed using GraphPad Prism (version 5) software. Statistical differences were determined by analysis of variance (ANOVA) followed by Tukey's multiple comparison test or Student's t-test where appropriate. Numerical and graphical results are displayed as mean \pm standard deviation. Significance was accepted at a level of $p<0.05$. Sample size $(n)$ is indicated within the corresponding figure legends.

\section{Results}

\subsection{Development of pore-forming bioinks}

To generate bioinks whose porosity increases post-printing, alginate and methylcellulose were mixed at alginate:methylcellulose weight ratios of 1:0, 1:1 and 1:2 (Fig. 1a) to form alginate-methylcellulose (ALG-MC) hybrid hydrogels. These composites formed stable hydrogels (Fig. 1b), but one day post-fabrication clearly differed in both their macro- (Fig. 1c) and microstructure (Fig. 1d) compared to solid alginate hydrogels. Methylcellulose (1:1 and 1:2) containing hydrogels showed larger pores than the alginate only gels (Fig. 1d and e), with higher mean pore diameter as the methylcellulose content was increased. This correlated with a faster reduction in the wet weight (w/w) (Fig. 1g) and a lower bulk Young's modulus (Fig. 1f) for the ALG-MC hybrid gels one day post-fabrication.

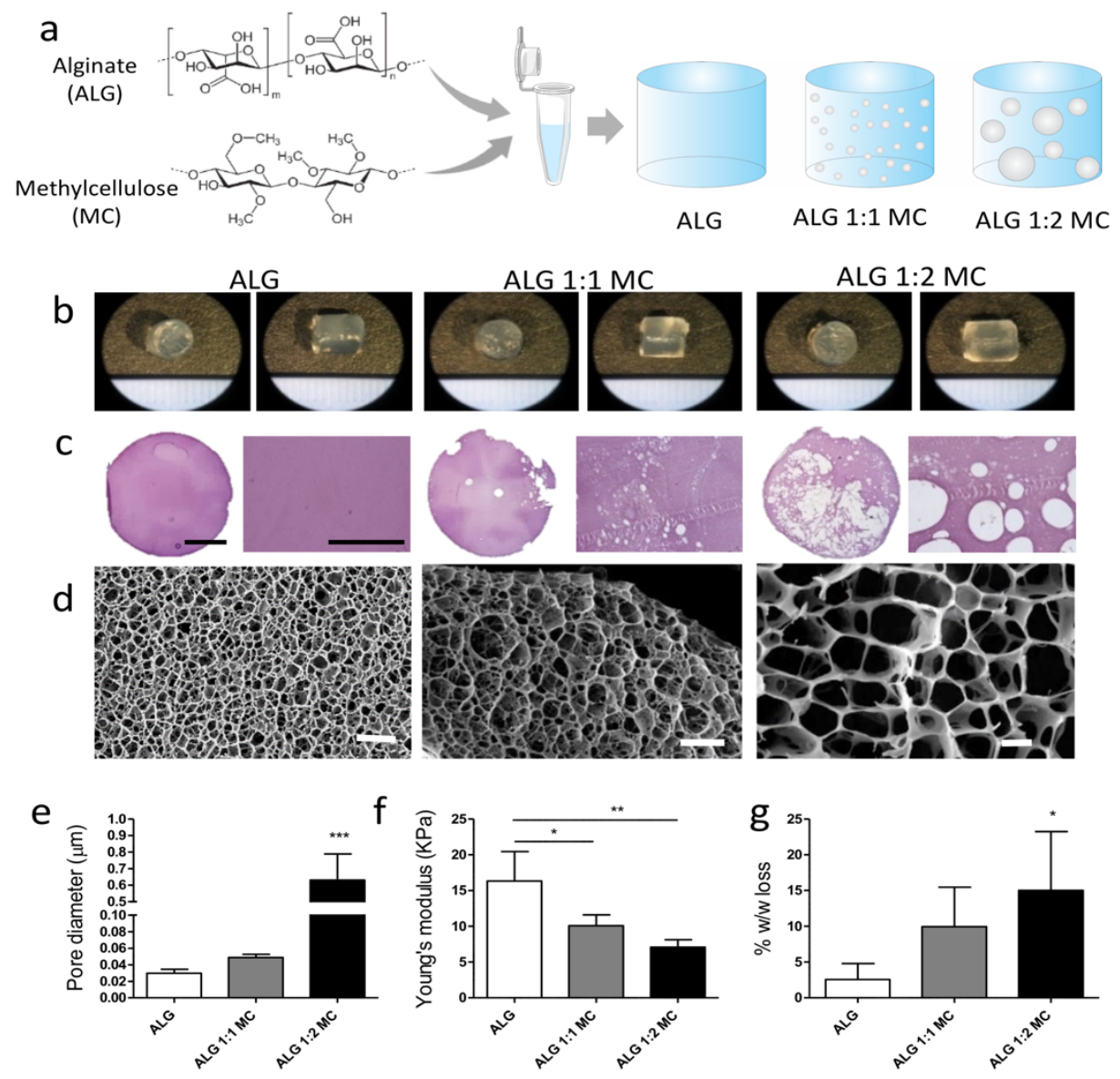


Figure 1. Development and characterization of pore-forming ALG-MC gels. a, Schematic of the fabrication of the ALG-MC gels. b, Macroscopic appearance (top and lateral view) of cylindrical gels fabricated with alginate and ALG-MC at 1:1 and 1:2 alginate-methylcellulose ratios. c, Histological representation through H\&E staining of the microscopic structure of the alginate and ALG-MC gels 1 day post-fabrication; scale bar $=1 \mathrm{~mm}$ in the lower magnification images and $0.2 \mathrm{~mm}$ in the higher magnification images. d, CryoSEM analysis of the micro-porosity of alginate and ALG-MC gels, scale bar = $1 \mu \mathrm{m}$. e, Pore diameter calculation based on the cryoSEM imaging of the alginate and alginate-MC gels. $\mathbf{f}$, Young's modulus of the alginate and ALG-MC gels 1 day post-fabrication. $\mathbf{g}$, Percentage of wet weight loss in the alginate and ALG-MC gels after 7 days in aqueous solution. $\left({ }^{*}\right)$ Denotes significance $(n=4, p<0.05)$ in comparison to the rest of the groups; ${ }^{*} p<0.01(n=4)$ and $* * * p<0.001(n=4)$ in comparison to the rest of the groups.

We next sought to assess how the incorporation of methylcellulose into alginate influenced the rheological behavior and printability of the resulting bioink. In agreement with previous studies[30,31], the addition of methylcellulose significantly increased the resulting viscosity compared to un-crosslinked alginate only, with the bioink exhibiting shear thinning properties (Fig. 2a) and thixotropic behavior (Fig. 2b). The printability of these pore-forming ALG-MC bioinks (1:2) was comparable to alginate pre-crosslinked with $\mathrm{CaCl}_{2}$ (ALG-Ca) (Fig. 2c-g), a strategy which is commonly used to improve the printability of alginate bioinks and to produce hydrogels for tissue engineering applications[7,9]. To confirm that such bioinks could be used to produce constructs with spatially defined gradients of cell populations, MSCs transfected with plasmid DNA (pDNA) encoding for GFP (green) and dtTomato (red) were encapsulated in ALG$\mathrm{Ca}$ and ALG-MC bioinks respectively (Fig. 2h). Both gels were simultaneously printed, using a multi-head printing system, in different patterns showing defined spatial distributions of both cell populations (Fig. 2i). To confirm that these pore-forming ALG-MC bioinks (1:2 weight ratio) could support stem cell differentiation, the viability and chondrogenic potential of MSCs in ALGMC and ALG-Ca bioinks post-printing were assessed (Supplementary Fig. 1a). The ALG-MC bioinks supported significantly higher levels of cell viability post-printing compared to the ALGCa bioinks (Supplementary Fig. 1b and c). Robust GAG (Supplementary Fig. 1e and h) and collagen type II deposition (Supplementary Fig. 1h) was observed in both bioinks, demonstrating that both inks were capable of supporting chondrogenesis of MSCs. Higher levels of calcification (Supplementary Fig. 1g and h) and collagen type X deposition (Supplementary Fig. 1h), both hallmarks of chondrocyte hypertrophy and endochondral progression, were observed in the alginate bioinks pre-crosslinked with $\mathrm{CaCl}_{2}$. This is likely due to the higher stiffness of these gels and the presence of calcium, which is known to be osteo-inductive[32,33]. 

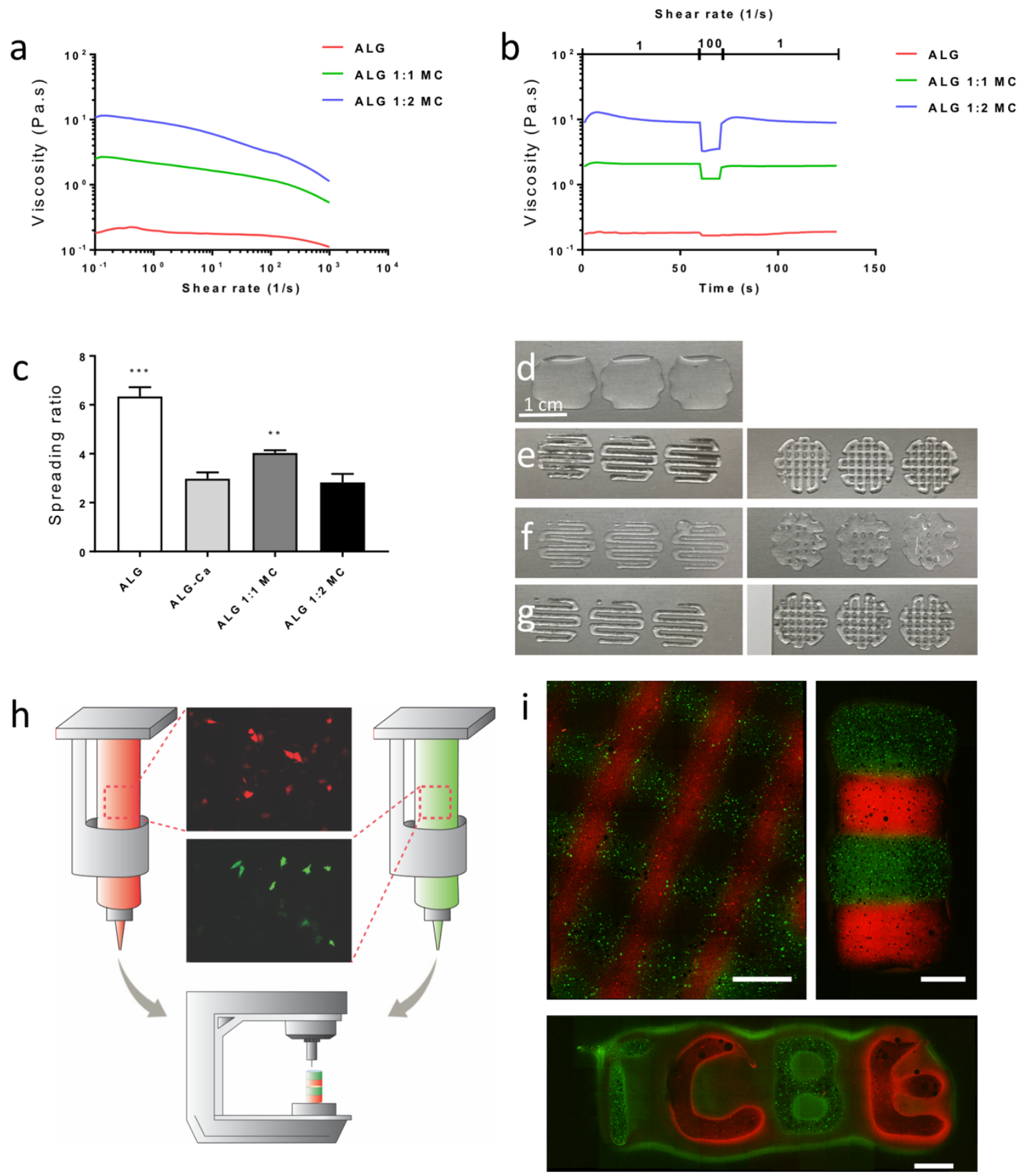

Figure 2. Printability of ALG-MC hybrid gels. a, Viscosity of ALG-Ca and ALG-MC gels (ALG 1:1 MC and ALG 1:2 MC) as a function of shear rate. $\mathbf{b}$, Viscosity recovery test as a function of shear rate. $\mathbf{c}$, Spreading ratio of the alginate, ALG-Ca and ALG-MC gels. Images of printed patterns of the alginate d, ALG-Ca e, ALG-MC 1:1 $\mathbf{f}$ and ALG-MC 1:2 $\mathbf{g}$, scale bar $=1 \mathrm{~cm}$. $\mathbf{h}$, Schematic of the printing of ALG-Ca gels containing GFP transfected MSCs and the ALG-MC 1:2 containing dtTomato transfected MSCs. i, 3D printed patterns with the ALG-Ca bioink containing GFP transfected cells and the ALG-MC 1:2 bioink containing dtTomato transfected cells, scale bar $=2 \mathrm{~mm}$. ${ }^{* *} p<0.01(n=4)$ and $* * * p<0.001(n=4)$ in comparison to the rest of the groups. 


\subsection{Pore-forming bioinks enhance nanoparticle-mediated reporter gene delivery in vitro}

Modulating the porosity of hydrogels through, for example ultrasound pulses, has previously been used to enable the on-demand release of encapsulated nanoparticle-pDNA complexes[34]. We hypothesized that our pore-forming bioinks could similarly lead to enhanced non-viral gene delivery in bioprinted constructs. CryOSEM was used to confirm that nanoparticle RALA peptide[25] (RALA)-pDNA complexes could be encapsulated in solid alginate and pore-forming (ALG-MC) hydrogels (Fig. 3a), with comparable encapsulation efficiencies observed in both systems (Fig. 3b). The rate of release of entrapped RALA-pDNA nanoparticle complexes was enhanced with increases in the concentration of methylcellulose within the hydrogel (Fig. 3c). When MSCs were co-encapsulated with RALA-pDNA complexes encoding for reporter genes within the hydrogels, sustained cell transfection was observed over 11 days suggesting continuous complex uptake (Supplementary Fig. 2a), with increased expression of the transgene observed in hydrogels with higher proportions of methylcellulose (Fig. 2c). Cell viability, indirectly assessed by measuring DNA content over time, was comparable in solid and poreforming ALG-MC hydrogels (Supplementary Fig. 2b). 

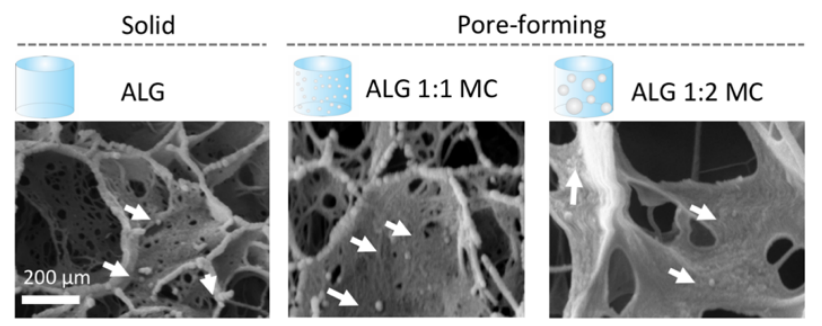

b

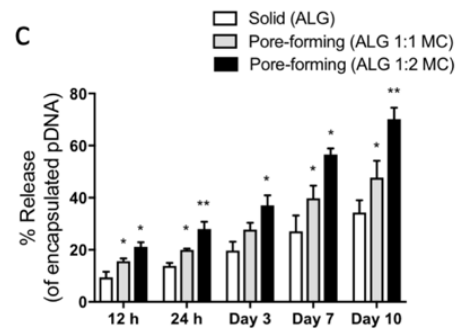

d
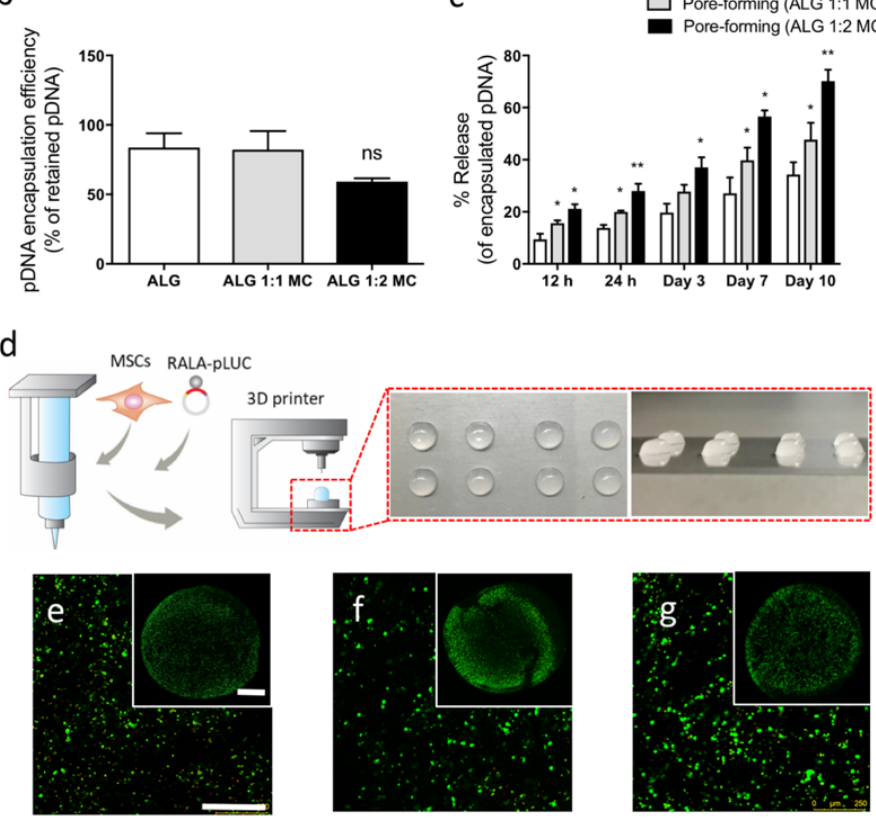

h
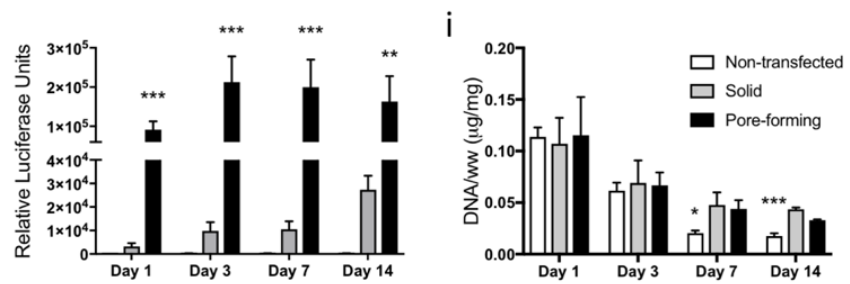

Figure 3. Characterization of gene activated pore-forming bioinks. a, CryoSEM images of the encapsulated RALA-pDNA complexes in the solid alginate and pore-forming ALG-MC (1:1 and 1:2) gels. Scale bar $=200 \mu \mathrm{m}$. White arrows point to RALA-pDNA complexes. $\mathbf{b}$, Encapsulation efficiency of the RALApDNA complexes in the different gels. c, Percentage of the released RALA-pDNA complexes in the different gels at 12 h, 24 h, day 3, 7 and 10. d, Schematic of cell-laden RALA-pLUC gene activated bioinks and printing, and macroscopic pictures of the 3D printed gene activated gels. Live/dead staining in the 3D printed e ALG-Ca non-transfected control, f solid bioinks (ALG-Ca) containing RALA-pLUC complexes, and g pore-forming bioinks (ALG 1:2 MC) containing RALA-pLUC complexes gels, 1 day after fabrication. Scale bar $=1 \mathrm{~mm}$ for the large magnification pictures and $250 \mu \mathrm{m}$ for the higher magnification. $\mathbf{h}$, Luciferase quantification in the culture media of the 3D printed non-transfected, solid and pore-forming gels containing RALA-pLUC complexes at day 1, 3, 7 and 14 after fabrication. i, DNA quantification in the nontransfected, solid and pore-forming 3D printed constructs containing RALA-pLUC complexes at day 1, 3, 7 and 14 after fabrication. $p>0.05(n=4), * p<0.05(n=4), * * p<0.01(n=4), * * * p<0.001(n=4)$ in comparison to the rest of the groups at the same time point. 
Delivery of reporter genes to encapsulated MSCs was also assessed in 3D bioprinted constructs. MSCs and RALA-pLUC complexes were encapsulated in either ALG-Ca (hereafter simply referred to as solid bioinks) or 1:2 ALG-MC (hereafter simply referred to as pore-forming bioinks) and printed to form disks (Fig. 3d). One day after printing, live/dead analysis demonstrated limited cell death in transfected constructs and non-transfected controls (Fig. 3e, $f$ and $g$ ). The DNA content within the printed gene activated constructs was also at least comparable to the non-transfected controls at all time-points, confirming that complex delivery had no long-term effects on cell viability (Fig. 3i). Importantly, luciferase gene expression was approximately 10-fold higher within the pore-forming bioinks compared to their solid counterparts, where a more gradual increase in expression was observed (Fig. 3h). Similar findings were made when an alternative nanoparticle-based gene delivery vector (specifically nanohydroxyapatite (nHA)[23]-pLUC complexes) was encapsulated into the pore-forming bioinks (Supplementary Fig. 3), confirming that the utility of these GABs is not limited to usage with the RALA peptide as the delivery vector and hence demonstrating their versatility.

\subsection{Pore-forming bioinks enhance nanoparticle-mediated gene delivery in vivo}

Having demonstrated that pore-forming bioinks enhance non-viral gene delivery in vitro, we next sought to evaluate their potential in vivo to transfect cells that were either co-printed into the construct or recruited following implantation into the body. To this end, acellular or MSC laden solid and pore-forming bioinks containing RALA-pLUC complexes were used to print cylindrical discs which were then implanted subcutaneously in nude mice (Fig. 4a). In vivo luciferase expression analysis revealed distinct expression profiles, with expression peaking early and decreasing more rapidly over time in the acellular and MSC laden pore-forming constructs, while a more sustained expression was observed in the solid constructs (Fig. 4). This was particularly pronounced when MSCs were directly printed into the constructs, where luciferase expression was an order of magnitude higher using the pore-forming bioinks at day 3 in comparison to the solid constructs (Fig. $4 b$ and d), with expression then decreasing rapidly with time (Fig. 4d). 


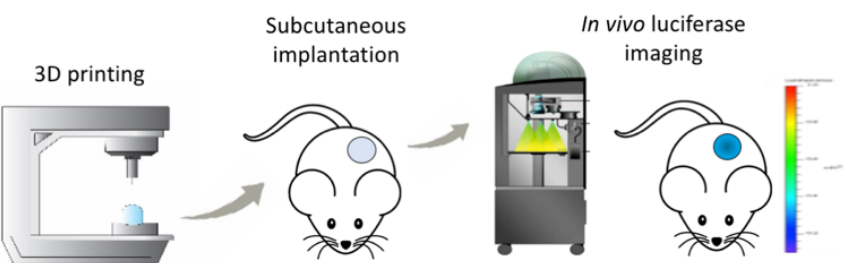

b

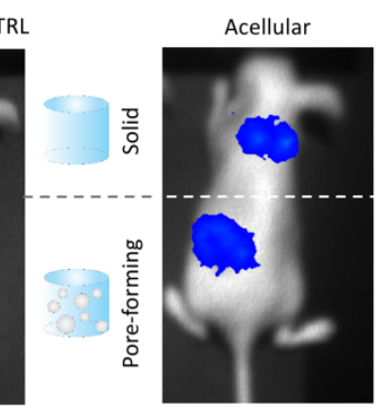

Cellular
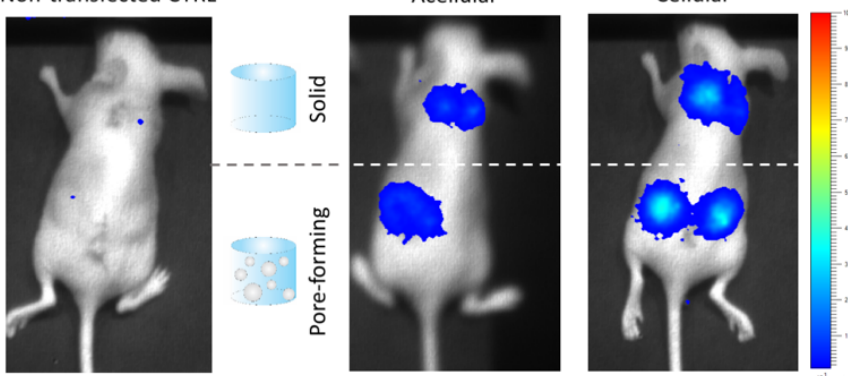

c

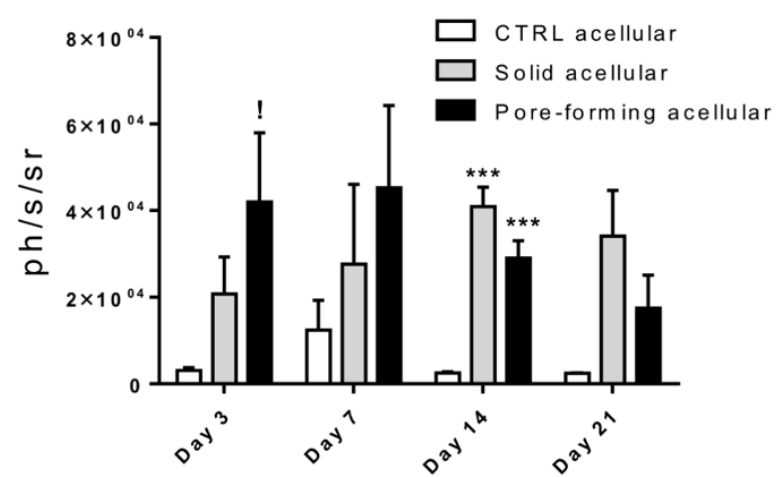

d

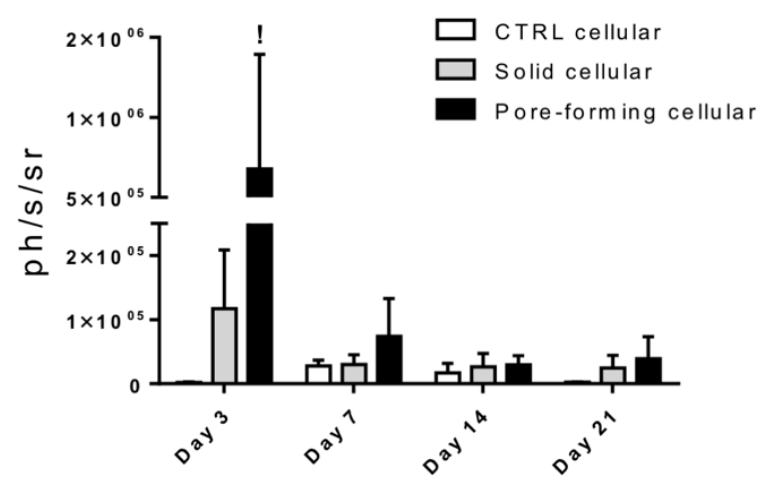

Figure 4. In vivo RALA-mediated gene delivery in 3D printed ALG-Ca and ALG-MC constructs. a, Schematic of 3D printing of alginate-based bioinks and mouse subcutaneous implantation to assess the in vivo luciferase expression. $\mathbf{b}$, Macroscopic images of the nude mice during the bioluminescence imaging protocol in the non-transfected control (top - CTRL acellular; bottom - CTRL cellular), acellular bioprinted tissues (top - Solid bioink; bottom - Pore-forming bioink), and cellular (MSC-laden) bioprinted tissues (top - Solid bioink; bottom - Pore-forming bioink) 3 days after implantation. Bioluminescence quantification $(\mathrm{ph} / \mathrm{s} / \mathrm{sr})$ of the acellular $\mathrm{c}$ and cell laden $\mathbf{d}$ gene activated gels. ! $\mathrm{p}<0.05(\mathrm{n}=6)$ in comparison to the nontransfected control (CTRL) at the same time point; ${ }^{* * *} p<0.001(n=>4)$ in comparison to the rest of the groups at the same time point. 


\subsection{Therapeutic gene delivery within bioprinted constructs can direct MSC fate in vitro}

and in vivo

We next sought to evaluate the capacity of these GABs to direct the differentiation of MSCs in vitro and in vivo. To promote chondrogenesis of MSCs, we selected the pore-forming bioinks using RALA as the delivery vector for different chondrogenic gene combinations ('TGF- $\beta 3$ and BMP2' or 'TGF- $\beta 3$, BMP2 and SOX9') based on previous data from our lab supporting the use of the RALA vector for cartilage tissue engineering[35]. To promote osteogenesis of MSCs, we selected the solid bioinks as these were previously shown to support an osteogenic/endochondral phenotype (Supplementary Fig.1g), with nHA as the gene delivery vector for BMP-2, based on previous data demonstrating the osteo-inductivity of these nanoparticles and their potential for gene delivery $[23,24,35,36]$. The co-delivery of TGF- $\beta 3$ and BMP2 genes to promote chondrogenesis of MSCs was based in previous studies from our research group[35,37], while the addition of SOX9 was motivated to suppress the tendency of MSCs to undergo hypertrophy and progress along an endochondral pathway[38-40]. Bioinks containing MSCs and vector-pDNA complexes were 3D printed and maintained in vitro for four weeks. Measurements of BMP2 and TGF- $\beta 3$ levels in the media confirmed that RALA and $\mathrm{nHA}$ were able to successfully deliver the pDNA to encapsulated MSCs and promote protein production (Supplementary Fig. 4). nHA-mediated delivery of pBMP2 promoted in vitro calcification (Fig. 5g, and $\mathrm{i}$ ), and enhanced mineralization and vascularization in vivo (Fig. $5 \mathrm{j}$ and $\mathrm{k}$ ). In contrast, the RALA-mediated co-delivery of pTGF-pBMP and pTGF-pBMP-pSOX9 promoted chondrogenesis of MSCs in vitro and in vivo (Fig. $5 \mathrm{~g}, \mathrm{f}, \mathrm{i}$ and $\mathrm{k}$ ). While both chondrogenic gene combinations were shown to promote GAG and collagen type II deposition, pTGF-pBMP co-delivery also promoted collagen type I deposition in vitro (Fig. $5 \mathrm{i}$ ) and collagen type $\mathrm{X}$ production in vivo (Fig. 5k), suggesting the progression of bioprinted MSCs along an endochondral pathway[41], while the co-delivery of SOX9, BMP2 and TGF- $\beta 3$ promoted the development of a more stable hyaline cartilage phenotype (Fig. $5 \mathrm{j}$ and I). 

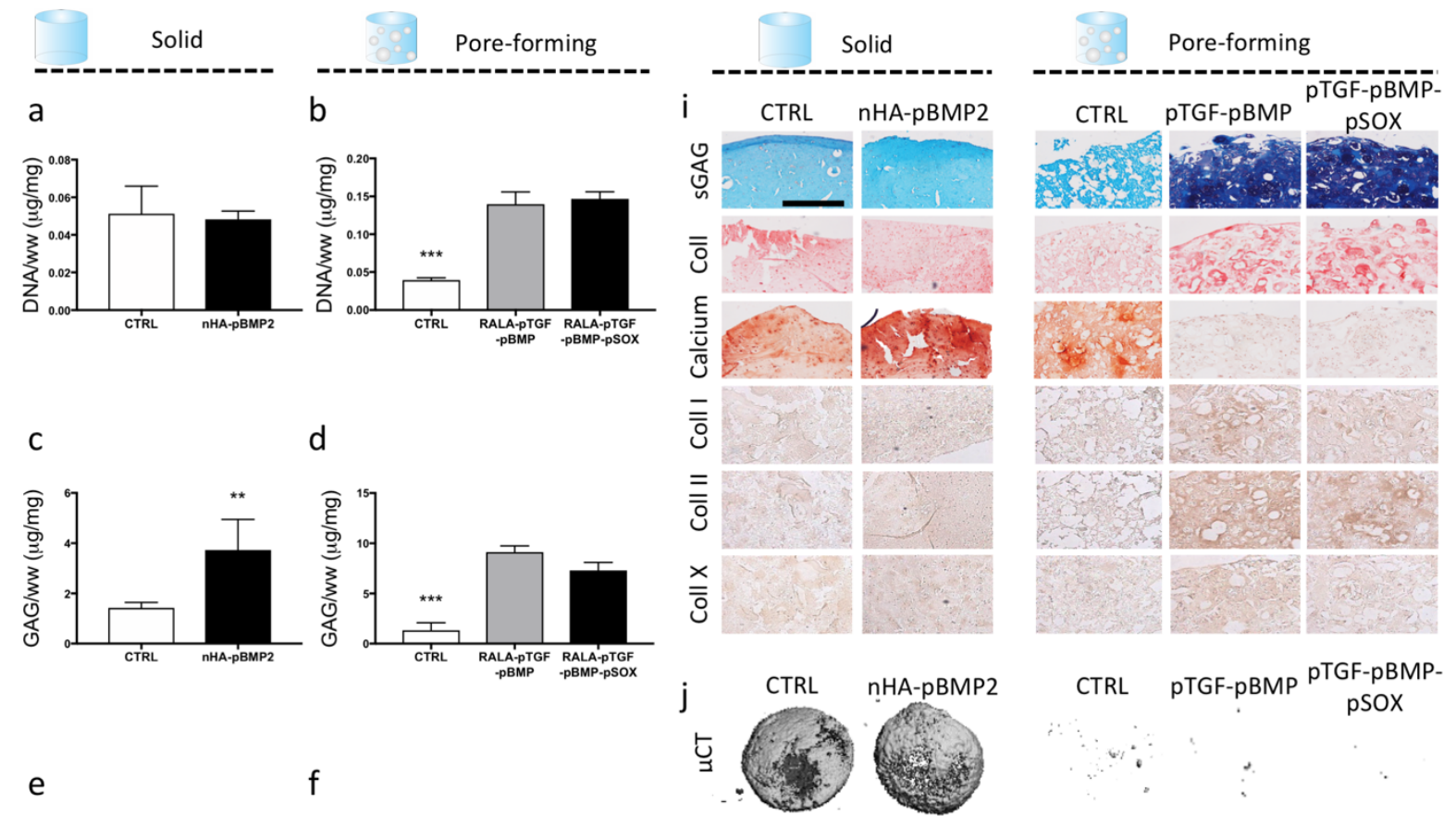

d
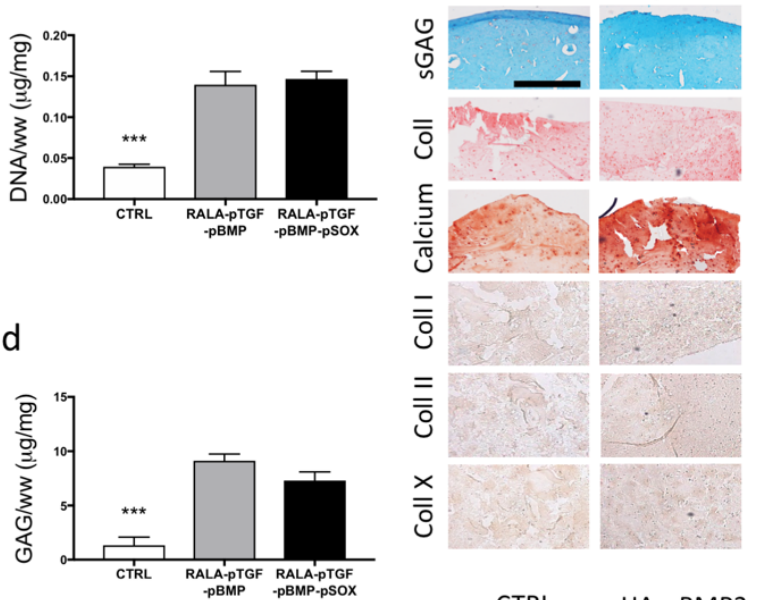

$\overline{\overline{0}}$

$\overline{\overline{0}}$

$\stackrel{\times}{\overline{0}}$
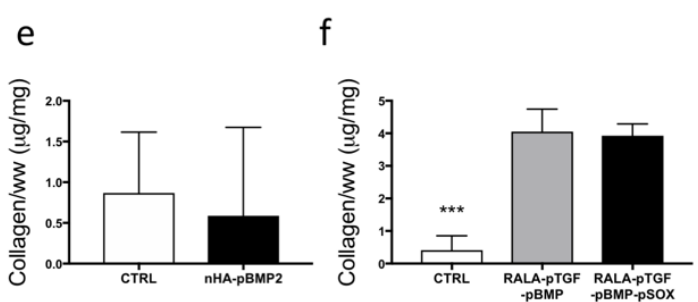

j
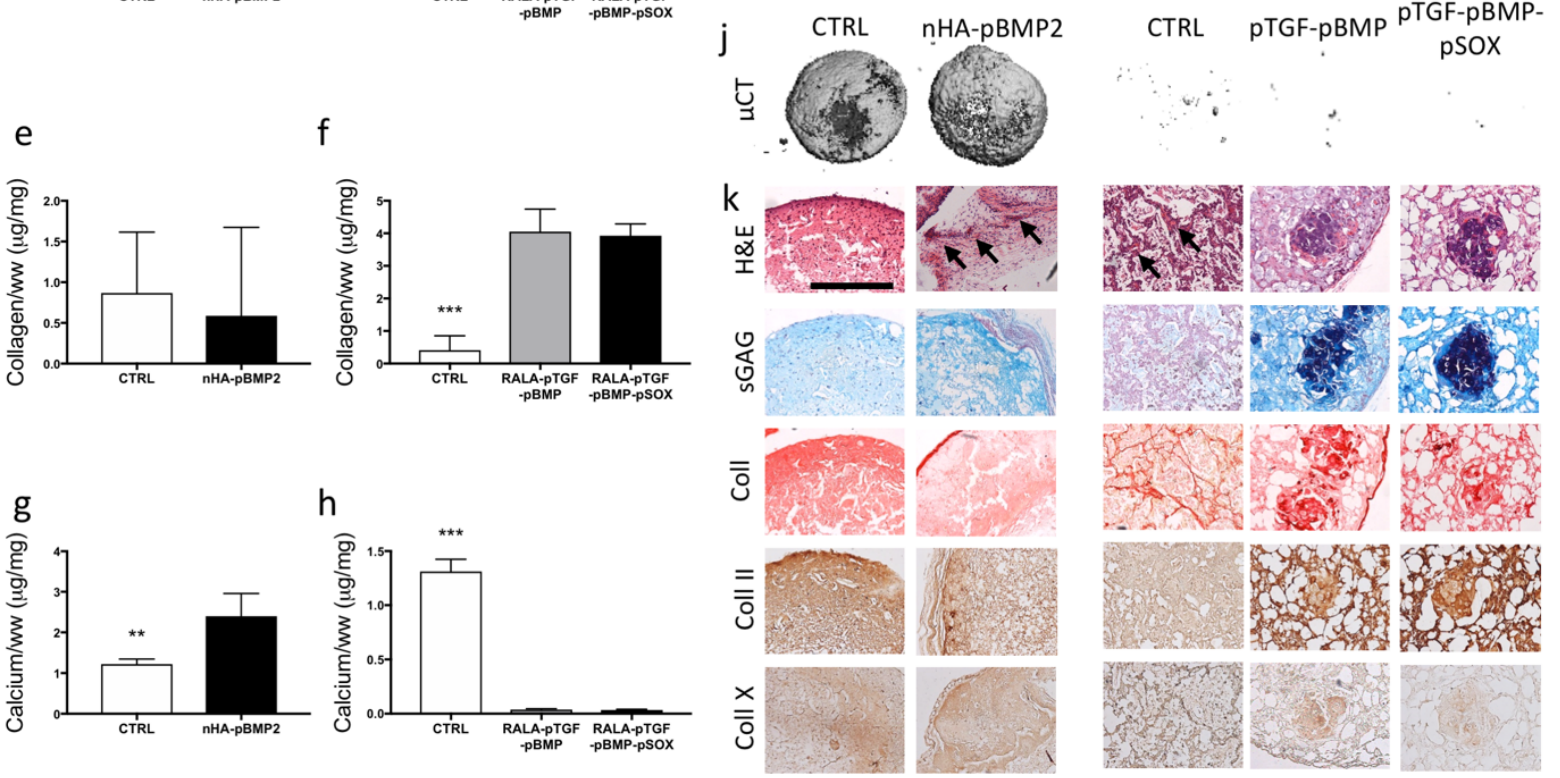

Figure 5. In vitro and in vivo therapeutic gene delivery in 3D printed gels for stem cell chondrogenesis

and osteogenesis. Quantification of $\mathbf{a}, \mathbf{b}$ DNA, $\mathbf{c}, \mathbf{d}$ GAG, $\mathbf{e}, \mathbf{f}$ collagen, and $\mathbf{g}$, $\mathbf{h}$ calcium deposition after 28 days of in vitro culture in solid and pore-forming $3 \mathrm{D}$ printed hydrogels containing no pDNA (CTRL) and either nHA-pBMP2 or RALA complexed to pTGF- $\beta 3$ and pBMP2 (pTGF-pBMP) or pTGF- $\beta 3$, pBMP2 and pSOX9 (pTGF-pBMP-pSOX9). i, Histological and immuhistochemical examination of the control and gene activated constructs after 28 days of in vitro culture. Scale bar $=0.5 \mathrm{~mm}$. j, MicroCT images of the implanted groups after four weeks in vivo. Scale bar $=1 \mathrm{~mm}$. $\mathbf{k}$, Histological and immunohistochemical examination of the implanted constructs after four weeks in vivo. Black arrows highlight areas of blood vessel activity. Scale bar $=0.5 \mathrm{~mm} .{ }^{* *} p<0.01(n=5), * * * p<0.001(n=5)$ in comparison to the rest of the groups. 


\subsection{Spatial deposition of gene activated bioinks to zonally direct stem cell fate}

As a proof-of-concept for the application of these gene activated bioinks to spatially direct stem cell differentiation, we next sought to engineer a mechanically reinforced, bilayered construct for the recapitulation of the osteochondral unit. Cylindrical porous polycaprolactone $(\mathrm{PCL})$ constructs were 3D printed with bulk mechanical properties similar to those of articular cartilage (Supplementary Figure 5a-d) to provide a level of mechanical functionality compatible with load bearing locations in vivo[7]. Depositing the bioinks within these printed PCL scaffolds did not negatively impact their capacity to transfect MSCs (Supplementary Figure 5e-g). The osseous region of these 3D printed constructs was then filled with either MSC laden, solid control (no plasmid) or gene activated (nHA - pBMP2 complexes) osteogenic bioinks. The cartilaginous region was filled with either MSC laden, pore-forming control (no plasmid) or a gene activated (RALA - pTGF-pBMP2-pSOX9) chondrogenic bioink.

In vitro, no differences in GAG (Fig. 6a and d), collagen (Fig. 6a and e) or calcium (Fig. 6a and f) deposition was observed between the cartilage and bone region of the control constructs. In contrast, the cartilage layer of the gene activated construct contained significantly higher levels of GAG (Fig. 6b and d) and collagen (Fig. 6b and f), and lower levels of calcium (Fig. 6b and f), in comparison to their corresponding bone layer and both layers of the control groups. Higher levels of collagen type II deposition were also observed in the cartilage layers of the gene activated constructs (Fig. 6g), with no evidence of significant collagen type I or X deposition. 


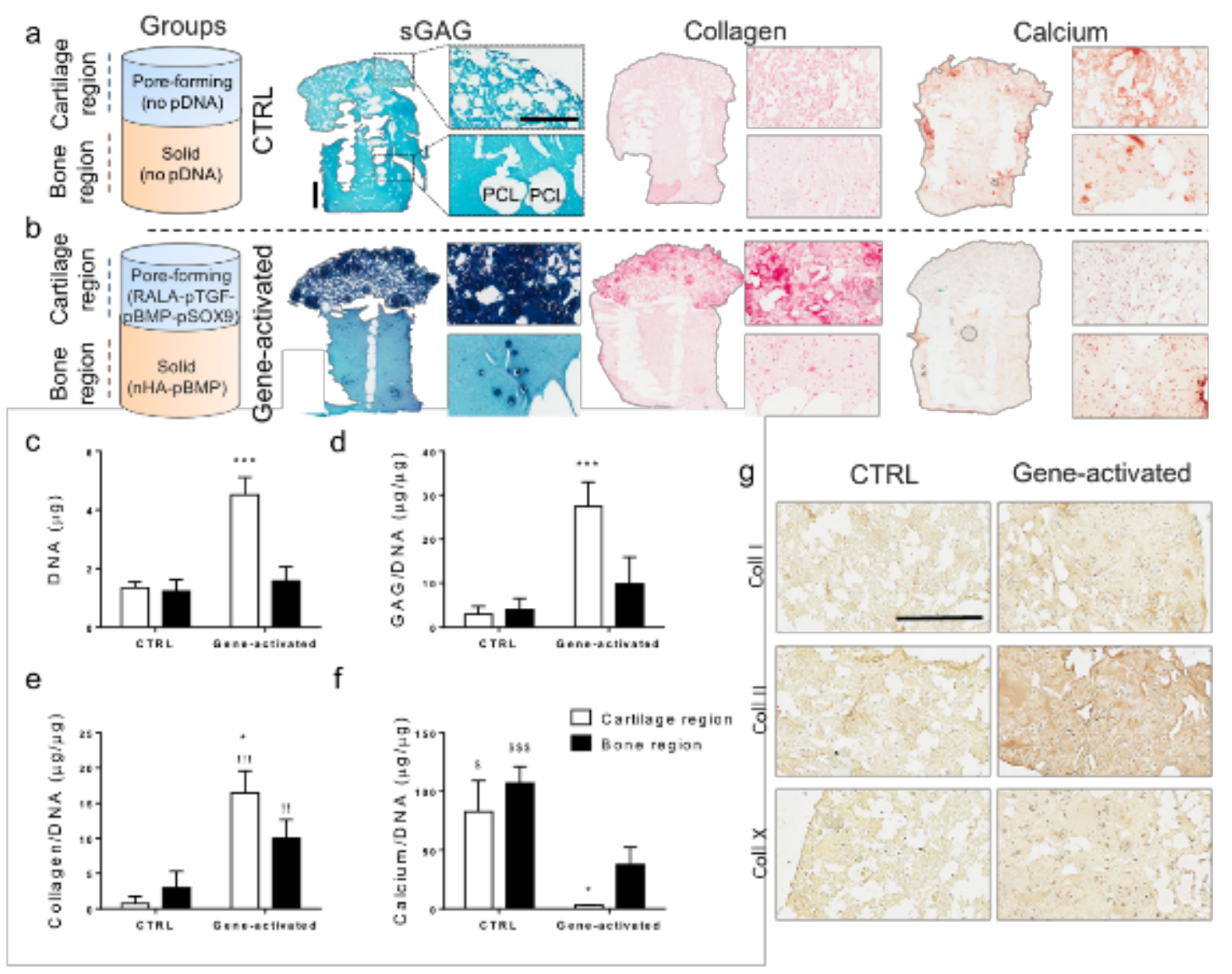

Figure 6. Spatial therapeutic gene delivery in mechanically reinforced bioinks in vitro. Histological analysis of GAG, collagen and calcium of the $\mathbf{a}$ control and $\mathbf{b}$ gene activated (nHA-pBMP/RALA-pTGFpBMP-pSOX9) groups after 28 days of in vitro culture. Quantification of levels of DNA c, GAG/DNA d, collagen/DNA $\mathbf{e}$ and calcium/DNA $\mathbf{f}$ in the cartilage and bone layers of the control and gene activated groups after 28 days of in vitro culture. $\mathbf{g}$, Immunohistochemical analysis of the cartilage region of the control and gene activated bioinks. Scale bar $=1 \mathrm{~mm}$ in the high magnification images and $0.2 \mathrm{~mm}$ in the rest of the images. ${ }^{*} p<0.05(n=4), * * * p<0.001(n=4)$ in comparison to the rest of the groups; !! p < $0.01(n=4)$, !! $p<0.001(n=4)$ in comparison to the CTRL groups; $\$ p<0.01(n=4), \$ \$ \$ p<0.001(n=4)$ in comparison to the gene activated groups.

In order to assess the potential of these constructs to spatially direct MSC differentiation in vivo, control and gene activated constructs were next implanted subcutaneously in nude mice. After four weeks in vivo, evidence of mineralization was observed in the bone and cartilage phases of the control implants (Fig. 7a and c). In contrast, little to no mineralization was observed in the cartilage layer of gene activated constructs (Fig. 7b and c), and instead mineralization was spatially restricted to the bone region of these implants. Higher levels of vascularization was observed in the bone layers of gene activated constructs (Fig. 7b and e) compared to gene-free controls (Fig. 7a and e). Cellular aggregates within the cartilage layer of 
gene activated constructs also stained positively for GAG (Fig. 7b) and collagen type II (Fig. 7e) deposition, and negatively for collagen type X (Fig. 7f).

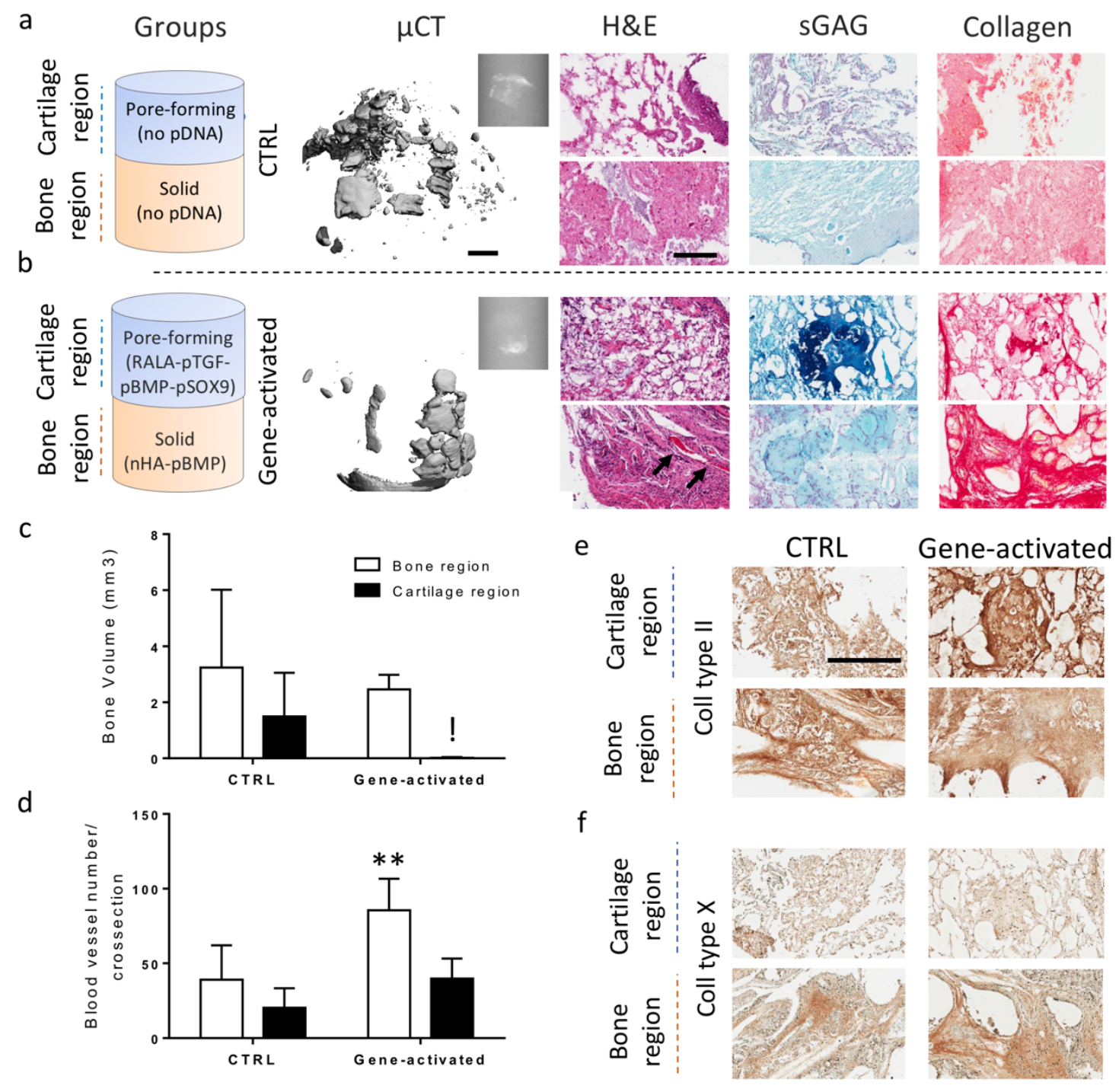

Figure 7. Spatial therapeutic gene delivery in mechanically reinforced bioinks in vivo. MicroCT, Histological analysis of H\&E, GAG, collagen and calcium staining of the $\mathbf{a}$ control, and $\mathbf{b}$ gene activated (nHApBMP/RALA-pTGF-pBMP-pSOX9) groups after four weeks of in vivo implantation. Black arrows highlight areas of blood vessel activity. Quantification of levels of $\mathbf{c}$ bone volume and $\mathbf{d}$ blood vessels in the cartilage and bone layers of the control and gene activated groups after four weeks of in vivo implantation. Immunohistochemical analysis of the cartilage and bone layers $\mathbf{e}$ collagen type II and $\mathbf{f}$ type $\mathrm{X}$ content of the control and gene activated bioinks after four weeks of in vivo implantation. Scale bar $=1 \mathrm{~mm} .{ }^{* *} \mathrm{p}<$ $0.01(n=>4)$ in comparison to all the groups; ! $p<0.05(n=>4)$, in comparison to the osteo layers of the control and gene activated groups. 


\section{Discussion}

The overall objective of this study was to develop a bioprinting strategy to enable spatiotemporally defined gene delivery to stem cells for the engineering of complex tissues. To spatially and temporally tune gene delivery in bioprinted tissues, a pore-forming bioink was first developed by blending alginate and methylcellulose, which enabled accelerated release of nonviral vector-pDNA complexes and enhanced the early transfection of encapsulated MSCs both in vitro and in vivo. When used to deliver an appropriate combination of chondrogenic genes (pTGF-pBMP-pSOX9) to MSCs, these pore-forming bioinks were able to support robust and stable chondrogenesis of MSCs in vitro and in vivo. To demonstrate the utility of this spatiotemporal control of gene delivery for the bioprinting of complex tissues, pro-osteogenic (solid bioinks containing nHA-pBMP-2) and pro-chondrogenic (pore-forming bioinks containing RALApTGF-pBMP-pSOX9) bioinks were spatially deposited into a 3D printed PCL scaffold, forming bilayered constructs able to zonally direct the differentiation of encapsulated MSCs. In vivo, the top pro-chondrogenic region of the bioprinted tissue supported stable chondrogenesis, with vascularization and mineralization generally confined to the bottom pro-osteogenic layer, thereby recapitulating key aspects of the gradients present in the native osteochondral unit.

Our bioprinting strategy relied not only on the ability to spatially deposit different gene and cell laden bioinks in 3D, but also on the capacity to temporally regulate pDNA delivery through the use of pore-forming bioinks. The addition of methylcellulose as a sacrificial material to develop a hybrid multicomponent gel allowed for increased printability and also, due to the lack of gelation of the methylcellulose component $[30,42,43]$ and its high solubility in aqueous solutions[43], permitted for its release into the surrounding media post-printing, resulting in dramatic changes to the hydrogel microstructure and micro-porosity. Increasing concentrations of methylcellulose resulted in larger pores, higher degradation rates and lower mechanical properties of the hybrid constructs. Nanoparticle-based gene delivery vectors, such as RALA and $\mathrm{nHA}$, have been previously explored by our research group for their incorporation into 3D materials to facilitate sustained delivery and enhanced therapeutic potential[35,36,44]. Although entrapment of nanoparticle-pDNA complexes into 3D printable hydrogels represents an opportunity to finely localize their delivery[36,45], their release and availability, especially of complexes larger than $10 \mathrm{~nm}$ [46], to transplanted or host cells can be limited[46]. This is due to the slow degradation kinetics of printable high molecular weight alginate and average pore sizes in the $n m$ range[12,46,47]. In the current study, the addition of methylcellulose to gene activated alginate gels enhanced the release of nanoparticle-pDNA complexes over time, facilitating accelerated complex uptake and reporter gene expression by encapsulated MSCs in 
comparison to solid non-porous gels. This enhanced release and transfection is likely due to the higher pore diameter of around $700 \mathrm{~nm}$ in the methylcellulose containing group, which will facilitated the uptake of RALA-pDNA and nHA-pDNA complexes whose size has been reported to be $60 \mathrm{~nm}[25,35]$ and $200 \mathrm{~nm}[23,24,35]$ respectively. In contrast, nanoparticle-pDNA complex encapsulation in solid alginate gels, which possess a pore size of around $20 \mathrm{~nm}$, offered a slower and more sustained release and MSC transfection overtime. Similar patterns of transfection were observed both in vitro and in vivo.

The engineering of phenotypically stable articular cartilage using bone marrow derived MSCs is challenging, as such cell source possesses an inherent tendency to progress along the endochondral pathway to form bone in vivo[7,29,48-52]. However, we found that the rapid delivery of pDNA encoding for TGF- $\beta 3$, BMP 2 and SOX9 in pore-forming constructs led to robust and stable chondrogenesis of MSCs in vitro and in vivo. The delivery of SOX9, a key transcription factor that inhibits RUNX2 transactivation and thus endochondral progression[39,53-55], together with the potent chondrogenic growth factors TGF- $\beta 3$ and BMP2, represents a combination of genes with the potential for inducing robust chondrogenesis of MSCs while supressing hypertrophy. Chondrogenesis within the pore-forming bioinks might be also enhanced by the lower stiffness of these gels[56,57] and their capacity to support MSC aggregation, as evident in the histological examination after in vivo implantation, which is critical for chondrogenic induction of MSCs[58,59].

To leverage the osteogenic potential of the solid alginate bioink, nHA-mediated gene delivery of BMP2 was incorporated to this system to promote bone formation. nHA-pBMP2 containing 3D printed hydrogels promoted calcium deposition in vitro, while in vivo they supported vascularization and mineralization of the gene activated constructs. A more sustained pBMP2 release and transfection, as provided by the solid bioinks, is believed preferable for bone regeneration, as recent studies suggest that more sustained recombinant BMP2 protein delivery from alginate hydrogels facilitates increased bone formation and reduces heterotrophic ossification in comparison to high-release kinetics[60]. $\mathrm{nHA}$ was chosen as gene delivery vector for our bone-forming bioink due to its osteoinductivity[61], higher bone regeneration potential in vivo in comparison to other non-viral vectors such as polyethilenimine (PEI)[62], and capacity to promote MSC hypertrophy and osteogenesis in vitro[35].

To demonstrate the utility of this bioprinting strategy, we engineered a gene activated, mechanically reinforced, bi-layered construct for the recapitulation of the osteochondral unit[63]. To engineer the articular cartilage region, we rapidly delivered RALA complexed to 
pTGF-pBMP-pSOX in pore-forming bioinks, while the underlying osseous region was created by delivering $\mathrm{nHA}$ complexed to $\mathrm{pBMP2}$ from solid bioinks. 3D printed PCL constructs, with bulk mechanical properties similar to those of articular cartilage, were used as scaffolding for the spatial distribution of the gene activated bioinks. After in vivo implantation, vascularization and mineralization were confined to the osseous region of gene activated implants, while in the cartilage region SGAG and type II collagen rich cell clusters were formed, demonstrating the potential of this system for spatially guiding stem cell differentiation and ECM deposition.

\section{Conclusion}

These results highlight the potential of bioink-guided gene delivery to engineer spatially complex musculoskeletal tissues, such as the osteochondral unit, that could be of clinical relevance for the treatment of injuries and disorders involving disruption of biological interfaces. This bioprinting strategy provides distinct advantages over current tissue engineering approaches and could be applied to the regeneration of a wide range of complex tissues; specifically, it allows for both temporally and spatially controlled delivery of therapeutic genes to host or implanted cells inside constructs with zonally definable mechanical properties. In turn, this enables the fine spatial control of stem cell differentiation and extracellular matrix development. Additionally, the combination of gene activated bioinks with multi-tool fabrication strategies might allow for the engineering of mechanically functional composites for a range of applications in orthopedic medicine and beyond.

Data availability. The authors declare that all data supporting the findings of this study are available within the paper and its Supplementary Information.

Acknowledgements. Funding from Science Foundation Ireland through the Advanced Materials and Bioengineering Research (AMBER) center and an Investigator Programme grant (12/IA/1554), as well as through the European Research Council (StemRepair, 258463; Joint Print, 647004). Valeria Nicolosi and Christopher Hobbs would like to thank the ERC (StG 2DNanocaps) and SFI (PIYRA and AMBER) for their support and the Advanced Microscopy Lab (AML) for the provision of their facilities. The authors would like to thank Prof. Matthias Möbius and Dr. Benjamin Haffner from the Trinity College Dublin School of Physics for the provision of their facilities and equipment.

\section{Disclosure}

No competing financial interests exist. 


\section{References}

[1] J.H. Shim, J.S. Lee, J.Y. Kim, D.W. Cho, Bioprinting of a mechanically enhanced threedimensional dual cell-laden construct for osteochondral tissue engineering using a multi-head tissue/organ building system, J. Micromechanics Microengineering. 22 (2012). doi:10.1088/0960-1317/22/8/085014.

[2] R. Levato, W.R. Webb, I.A. Otto, A. Mensinga, Y. Zhang, M. van Rijen, R. van Weeren, I.M. Khan, J. Malda, The bio in the ink: cartilage regeneration with bioprintable hydrogels and articular cartilage-derived progenitor cells, Acta Biomater. 61 (2017) 4153. doi:10.1016/j.actbio.2017.08.005.

[3] D.B. Kolesky, K.A. Homan, M.A. Skylar-Scott, J.A. Lewis, Three-dimensional bioprinting of thick vascularized tissues., Proc. Natl. Acad. Sci. U. S. A. 113 (2016) 3179-84. doi:10.1073/pnas.1521342113.

[4] N.E. Fedorovich, H.M. Wijnberg, W.J.A. Dhert, J. Alblas, Distinct Tissue Formation by Heterogeneous Printing of Osteo- and Endothelial Progenitor Cells, Tissue Eng. Part A. 17 (2011) 2113-2121. doi:10.1089/ten.tea.2011.0019.

[5] A.D. Graham, S.N. Olof, M.J. Burke, J.P.K. Armstrong, E.A. Mikhailova, J.G. Nicholson, S.J. Box, F.G. Szele, A.W. Perriman, H. Bayley, High-Resolution Patterned Cellular Constructs by Droplet-Based 3D Printing, Sci. Rep. 7 (2017). doi:10.1038/s41598-01706358-x.

[6] W. Schuurman, T.J. Klein, W.J.A. Dhert, P.R. van Weeren, D.W. Hutmacher, J. Malda, Cartilage regeneration using zonal chondrocyte subpopulations: a promising approach or an overcomplicated strategy?, J. Tissue Eng. Regen. Med. 9 (2015) 669-678. doi:10.1002/term.1638.

[7] A.C. Daly, G.M. Cunniffe, B.N. Sathy, O. Jeon, E. Alsberg, D.J. Kelly, 3D Bioprinting of Developmentally Inspired Templates for Whole Bone Organ Engineering, Adv. Healthc. Mater. 5 (2016) 2353-2362. doi:10.1002/adhm.201600182.

[8] D. Nguyen, D.A. Hgg, A. Forsman, J. Ekholm, P. Nimkingratana, C. Brantsing, T. Kalogeropoulos, S. Zaunz, S. Concaro, M. Brittberg, A. Lindahl, P. Gatenholm, A. Enejder, S. Simonsson, Cartilage Tissue Engineering by the 3D Bioprinting of iPS Cells in a Nanocellulose/Alginate Bioink, Sci. Rep. 7 (2017). doi:10.1038/s41598-017-00690-y.

[9] F.E. Freeman, D.J. Kelly, Tuning Alginate Bioink Stiffness and Composition for Controlled 
Growth Factor Delivery and to Spatially Direct MSC Fate within Bioprinted Tissues, Sci. Rep. 7 (2017) 1-12. doi:10.1038/s41598-017-17286-1.

[10] C.H. Lee, J.L. Cook, A. Mendelson, E.K. Moioli, H. Yao, J.J. Mao, Regeneration of the articular surface of the rabbit synovial joint by cell homing: a proof of concept study., Lancet. 376 (2010) 440-8. doi:10.1016/S0140-6736(10)60668-X.

[11] C.H. Lee, S.A. Rodeo, L.A. Fortier, C. Lu, C. Erisken, J.J. Mao, Protein-releasing polymeric scaffolds induce fibrochondrocytic differentiation of endogenous cells for knee meniscus regeneration in sheep, Sci. Transl. Med. 6 (2014). doi:10.1126/scitranslmed.3009696.

[12] A.C.A.C.A.C. Daly, F.E.F.E. Freeman, T. Gonzalez-Fernandez, S.E.S.E. Critchley, J. Nulty, D.J.D.J.D.J. Kelly, 3D Bioprinting for Cartilage and Osteochondral Tissue Engineering, Adv. Healthc. Mater. 6 (2017). doi:10.1002/adhm.201700298.

[13] X. Huang, C.S. Brazel, On the importance and mechanisms of burst release in matrixcontrolled drug delivery systems, J. Control. Release. 73 (2001) 121-136. doi:10.1016/S0168-3659(01)00248-6.

[14] F.M. Chen, M. Zhang, Z.F. Wu, Toward delivery of multiple growth factors in tissue engineering, Biomaterials. 31 (2010) 6279-6308. doi:10.1016/j.biomaterials.2010.04.053.

[15] C.J. Kearney, D.J. Mooney, Macroscale delivery systems for molecular and cellular payloads, Nat. Mater. 12 (2013) 1004-1017. doi:10.1038/nmat3758.

[16] C. Evans, Using genes to facilitate the endogenous repair and regeneration of orthopaedic tissues., Int. Orthop. 38 (2014) 1761-9. doi:10.1007/s00264-014-2423-x.

[17] E.J. Sheehy, T. Mesallati, T. Vinardell, D.J. Kelly, Engineering cartilage or endochondral bone: A comparison of different naturally derived hydrogels, Acta Biomater. 13 (2014) 245-253. doi:10.1016/j.actbio.2014.11.031.

[18] H.G. Merkus, Particle Size Measurements, 2009. doi:10.1007/978-1-4020-9016-5.

[19] H. Li, S. Liu, L. Lin, Rheological study on 3D printability of alginate hydrogel and effect of graphene oxide, Int. J. Bioprinting. 2 (2016). doi:10.18063/IJB.2016.02.007.

[20] A.C. Daly, S.E. Critchley, E.M. Rensock, D.J. Kelly, E.M. Rencsok, D.J. Kelly, A Comparison of Different Bioinks for 3D Bioprinting of Fibrocartilage and Hyaline Cartilage, 
Biofabrication. 8 (2016) 1-25. doi:10.1088/1758-5090/8/4/045002.

[21] D.P. Lennon, A.I. Caplan, Isolation of human marrow-derived mesenchymal stem cells., Exp. Hematol. 34 (2006) 1604-5. doi:10.1016/j.exphem.2006.07.014.

[22] G.M. Cunniffe, F.J. O'Brien, S. Partap, T.J. Levingstone, K.T. Stanton, G.R. Dickson, The synthesis and characterization of nanophase hydroxyapatite using a novel dispersantaided precipitation method, J. Biomed. Mater. Res. Part A. 95A (2010) 1142-1149. doi:10.1002/jbm.a.32931.

[23] C.M. Curtin, G.M. Cunniffe, F.G. Lyons, K. Bessho, G.R. Dickson, G.P. Duffy, F.J. O’Brien, Innovative collagen nano-hydroxyapatite scaffolds offer a highly efficient non-viral gene delivery platform for stem cell-mediated bone formation., Adv. Mater. 24 (2012) 74954. doi:10.1002/adma.201103828.

[24] I.M. Castaño, C.M. Curtin, G. Shaw, J. Mary Murphy, G.P. Duffy, F.J. O’Brien, A novel collagen-nanohydroxyapatite microRNA-activated scaffold for tissue engineering applications capable of efficient delivery of both miR-mimics and antagomiRs to human mesenchymal stem cells., J. Control. Release. 200 (2014) 42-51. doi:10.1016/j.jconrel.2014.12.034.

[25] H.O. McCarthy, J. McCaffrey, C.M. McCrudden, A. Zholobenko, A. a Ali, J.W. McBride, A.S. Massey, S. Pentlavalli, K.-H. Chen, G. Cole, S.P. Loughran, N.J. Dunne, R.F. Donnelly, V.L. Kett, T. Robson, Development and characterization of self-assembling nanoparticles using a bio-inspired amphipathic peptide for gene delivery., J. Control. Release. 189C (2014) 141-149. doi:10.1016/j.jconrel.2014.06.048.

[26] G.M. Cunniffe, T. Vinardell, J.M. Murphy, E.M. Thompson, A. Matsiko, F.J. O'Brien, D.J. Kelly, Porous decellularized tissue engineered hypertrophic cartilage as a scaffold for large bone defect healing, Acta Biomater. 23 (2015) 82-90. doi:10.1016/j.actbio.2015.05.031.

[27] B.A. Tannous, Gaussia luciferase reporter assay for monitoring of biological processes in culture and in vivo, Nat. Protoc. 4 (2009) 582-591. doi:10.1038/nprot.2009.28.

[28] E.J. Sheehy, C.T. Buckley, D.J. Kelly, Oxygen tension regulates the osteogenic, chondrogenic and endochondral phenotype of bone marrow derived mesenchymal stem cells., Biochem. Biophys. Res. Commun. 417 (2012) 305-10. doi:10.1016/j.bbrc.2011.11.105. 
[29] E.J. Sheehy, T. Vinardell, M.E. Toner, C.T. Buckley, D.J. Kelly, Altering the architecture of tissue engineered hypertrophic cartilaginous grafts facilitates vascularisation and accelerates mineralisation, PLoS One. 9 (2014). doi:10.1371/journal.pone.0090716.

[30] K. Schütz, A.-M. Placht, B. Paul, S. Brüggemeier, M. Gelinsky, A. Lode, Threedimensional plotting of a cell-laden alginate/methylcellulose blend: towards biofabrication of tissue engineering constructs with clinically relevant dimensions, J. Tissue Eng. Regen. Med. 4 (2015) n/a-n/a. doi:10.1002/term.2058.

[31] H. Li, Y.J. Tan, K.F. Leong, L. Li, 3D Bioprinting of Highly Thixotropic Alginate/Methylcellulose Hydrogel with Strong Interface Bonding, ACS Appl. Mater. Interfaces. 9 (2017) 20086-20097. doi:10.1021/acsami.7b04216.

[32] C.S.D. Lee, H.R. Moyer, R.A. Gittens I., J.K. Williams, A.L. Boskey, B.D. Boyan, Z. Schwartz, Regulating in vivo calcification of alginate microbeads, Biomaterials. 31 (2010) 4926-4934. doi:10.1016/j.biomaterials.2010.03.001.

[33] R. Aquino-Martínez, N. Artigas, B. Gámez, J. Luis Rosa, F. Ventura, J.J. Cray, Extracellular calcium promotes bone formation from bone marrow mesenchymal stem cells by amplifying the effects of BMP-2 on SMAD signalling, PLoS One. 12 (2017). doi:10.1371/journal.pone.0178158.

[34] N. Huebsch, C.J. Kearney, X. Zhao, J. Kim, C.A. Cezar, Z. Suo, D.J. Mooney, Ultrasoundtriggered disruption and self-healing of reversibly cross-linked hydrogels for drug delivery and enhanced chemotherapy, Proc. Natl. Acad. Sci. 111 (2014) 9762-9767. doi:10.1073/pnas.1405469111.

[35] T. Gonzalez-Fernandez, B.N.N. Sathy, C. Hobbs, G.M.M. Cunniffe, H.O.O. McCarthy, N.J.J. Dunne, V. Nicolosi, F.J.J. O’Brien, D.J.J. Kelly, Mesenchymal stem cell fate following non-viral gene transfection strongly depends on the choice of delivery vector, Acta Biomater. 55 (2017) 226-238. doi:10.1016/j.actbio.2017.03.044.

[36] G.M. Cunniffe, T. Gonzalez-Fernandez, A. Daly, B.N. Sathy, O. Jeon, E. Alsberg, D.J. Kelly, Three-Dimensional Bioprinting of Polycaprolactone Reinforced Gene Activated Bioinks for Bone Tissue Engineering, Tissue Eng. Part A. 23 (2017) 891-900. doi:10.1089/ten.tea.2016.0498.

[37] T. Gonzalez-Fernandez, E.G.E.G.E.G.E.G. Tierney, G.M.G.M. Cunniffe, F.J.F.J.F.J. O’Brien, D.J.D.J. Kelly, Gene Delivery of TGF- $\beta 3$ and BMP2 in an MSC-Laden Alginate Hydrogel for 
Articular Cartilage and Endochondral Bone Tissue Engineering, Tissue Eng. Part A. 22 (2016) 776-787. doi:10.1089/ten.tea.2015.0576.

[38] J.S. Park, H.N. Yang, D.G. Woo, S.Y. Jeon, K.-H. Park, SOX9 gene plus heparinized TGF- $\beta 3$ coated dexamethasone loaded PLGA microspheres for inducement of chondrogenesis of hMSCs., Biomaterials. 33 (2012) 7151-63. doi:10.1016/j.biomaterials.2012.06.023.

[39] J. Liao, N. Hu, N. Zhou, L. Lin, C. Zhao, S. Yi, T. Fan, W. Bao, X. Liang, H. Chen, W. Xu, C. Chen, Q. Cheng, Y. Zeng, W. Si, Z. Yang, W. Huang, Sox9 potentiates BMP2-induced chondrogenic differentiation and inhibits BMP2-induced osteogenic differentiation, PLoS One. 9 (2014) e89025. doi:10.1371/journal.pone.0089025.

[40] T. Hattori, C. Muller, S. Gebhard, E. Bauer, F. Pausch, B. Schlund, M.R. Bosl, A. Hess, C. Surmann-Schmitt, H. von der Mark, B. de Crombrugghe, K. von der Mark, , SOX9 is a major negative regulator of cartilage vascularization, bone marrow formation and endochondral ossification, Development. 137 (2010) 901-911. doi:10.1242/dev.045203.

[41] K. Iyama, Y. Ninomiya, B.R. Olsen, T.F. Linsenmayer, R.L. Trelstad, M. Hayashi, Spatiotemporal pattern of type $\mathrm{X}$ collagen gene expression and collagen deposition in embryonic chick vertebrae undergoing endochondral ossification, Anat. Rec. 229 (1991) 462-472. doi:10.1002/ar.1092290405.

[42] S. Thirumala, J. Gimble, R. Devireddy, Methylcellulose Based Thermally Reversible Hydrogel System for Tissue Engineering Applications, Cells. 2 (2013) 460-475. doi:10.3390/cells2030460.

[43] P.L. Nasatto, F. Pignon, J.L.M. Silveira, M.E.R. Duarte, M.D. Noseda, M. Rinaudo, Methylcellulose, a cellulose derivative with original physical properties and extended applications, Polymers (Basel). 7 (2015) 777-803. doi:10.3390/polym7050777.

[44] B.N. Sathy, D. Olvera, T. Gonzalez-Fernandez, G. Inne, M. Cunniffe, S. Pentlavalli, P. Chambers, O. Jeon, E. Alsberg, H.O. Mccarthy, N. Dunne, T.L. Haut, D. Gh, D.J. Kelly, RALA complexed a-TCP nanoparticle delivery to mesenchymal stem cells induces bone formation in tissue engineered constructs in vitro and in vivo, J. Mater. Chem. B. 5 (2017) 1753-1764. doi:10.1039/c6tb02881k.

[45] T. Gonzalez-Fernandez, D.J. Kelly, F.J. O'Brien, Controlled Non-Viral Gene Delivery in Cartilage and Bone Repair: Current Strategies and Future Directions, Adv. Ther. (2018) 
1800038. doi:10.1002/adtp.201800038.

[46] C.J. Kearney, H. Skaat, S.M. Kennedy, J. Hu, M. Darnell, T.M. Raimondo, D.J. Mooney, Switchable Release of Entrapped Nanoparticles from Alginate Hydrogels, Adv. Healthc. Mater. 4 (2015) 1634-1639. doi:10.1002/adhm.201500254.

[47] E. Alsberg, H.J. Kong, Y. Hirano, M.K. Smith, A. Albeiruti, D.J. Mooney, Regulating bone formation via controlled scaffold degradation, J Dent Res. 82 (2003) 903-908. doi:10.1177/154405910308201111.

[48] K. Pelttari, A. Winter, E. Steck, K. Goetzke, T. Hennig, B.G. Ochs, T. Aigner, W. Richter, Premature induction of hypertrophy during in vitro chondrogenesis of human mesenchymal stem cells correlates with calcification and vascular invasion after ectopic transplantation in SCID mice., Arthritis Rheum. 54 (2006) 3254-66. doi:10.1002/art.22136.

[49] M.B. Mueller, R.S. Tuan, Functional characterization of hypertrophy in chondrogenesis of human mesenchymal stem cells, Arthritis Rheum. 58 (2008) 1377-1388. doi:10.1002/art.23370.

[50] C.A. Hellingman, E.N.B. Davidson, W. Koevoet, E.L. Vitters, W.B. van den Berg, G.J.V.M. van Osch, P.M. van der Kraan, Smad Signaling Determines Chondrogenic Differentiation of Bone-Marrow-Derived Mesenchymal Stem Cells: Inhibition of Smad1/5/8P Prevents Terminal Differentiation and Calcification, Tissue Eng. Part A. 17 (2011) 1157-1167. doi:10.1089/ten.tea.2010.0043.

[51] T. Vinardell, E.J. Sheehy, C.T. Buckley, D.J. Kelly, A comparison of the functionality and in vivo phenotypic stability of cartilaginous tissues engineered from different stem cell sources., Tissue Eng. Part A. 18 (2012) 1161-70. doi:10.1089/ten.TEA.2011.0544.

[52] G.M. Cunniffe, T. Vinardell, E.M. Thompson, A.C. Daly, A. Matsiko, F.J. O’Brien, D.J. Kelly, Chondrogenically primed mesenchymal stem cell-seeded alginate hydrogels promote early bone formation in critically-sized defects, Eur. Polym. J. 72 (2015) 464472. doi:10.1016/j.eurpolymj.2015.07.021.

[53] L. Cao, F. Yang, G. Liu, D. Yu, H. Li, Q. Fan, Y. Gan, T. Tang, K. Dai, The promotion of cartilage defect repair using adenovirus mediated Sox9 gene transfer of rabbit bone marrow mesenchymal stem cells., Biomaterials. 32 (2011) 3910-20. doi:10.1016/j.biomaterials.2011.02.014. 
[54] T. Hattori, C. Muller, S. Gebhard, E. Bauer, F. Pausch, B. Schlund, M.R. Bosl, A. Hess, C. Surmann-Schmitt, H. von der Mark, B. de Crombrugghe, K. von der Mark, SOX9 is a major negative regulator of cartilage vascularization, bone marrow formation and endochondral ossification, Development. 137 (2010) 901-911. doi:10.1242/dev.045203.

[55] S. Yamashita, M. Andoh, H. Ueno-Kudoh, T. Sato, S. Miyaki, H. Asahara, Sox9 directly promotes Bapx1 gene expression to repress Runx2 in chondrocytes, Exp. Cell Res. 315 (2009) 2231-2240. doi:10.1016/j.yexcr.2009.03.008.

[56] J.S. Park, J.S. Chu, A.D. Tsou, R. Diop, Z. Tang, A. Wang, S. Li, The effect of matrix stiffness on the differentiation of mesenchymal stem cells in response to TGF-beta, Biomaterials. 32 (2011) 3921-3930. doi:10.1016/j.biomaterials.2011.02.019.

[57] R.O. Navarrete, E.M. Lee, K. Smith, S.L. Hyzy, M. Doroudi, J.K. Williams, K. Gall, B.D. Boyan, Z. Schwartz, Substrate stiffness controls osteoblastic and chondrocytic differentiation of mesenchymal stem cells without exogenous stimuli, PLoS One. 12 (2017). doi:10.1371/journal.pone.0170312.

[58] S. Ghosh, M. Laha, S. Mondal, S. Sengupta, D.L. Kaplan, In vitro model of mesenchymal condensation during chondrogenic development, Biomaterials. 30 (2009) 6530-6540. doi:10.1016/j.biomaterials.2009.08.019.

[59] N. V. Ghone, W.L. Grayson, Recapitulation of mesenchymal condensation enhances in vitro chondrogenesis of human mesenchymal stem cells, J. Cell. Physiol. (2012). doi:10.1002/jcp.24078.

[60] L. Krishnan, L.B. Priddy, C. Esancy, B.S. Klosterhoff, H.Y. Stevens, L. Tran, R.E. Guldberg, Delivery vehicle effects on bone regeneration and heterotopic ossification induced by high dose BMP-2, Acta Biomater. 49 (2017) 101-112. doi:10.1016/J.ACTBIO.2016.12.012.

[61] G.M. Cunniffe, C.M. Curtin, E.M. Thompson, G.R. Dickson, F.J. O’Brien, ContentDependent Osteogenic Response of Nanohydroxyapatite: An in Vitro and in Vivo Assessment within Collagen-Based Scaffolds, ACS Appl. Mater. Interfaces. 8 (2016) 23477-23488. doi:10.1021/acsami.6b06596.

[62] C.M. Curtin, E.G. Tierney, K. McSorley, S.-A. Cryan, G.P. Duffy, F.J. O’Brien, Combinatorial gene therapy accelerates bone regeneration: non-viral dual delivery of 
VEGF and BMP2 in a collagen-nanohydroxyapatite scaffold., Adv. Healthc. Mater. 4 (2015) 223-7. doi:10.1002/adhm.201400397.

[63] F. Martini, J. Nath, E. Bartholomew, Fundamentals of anatomy and physiology, 2009. doi:612. 
Supplementary Material
Click here to download Supplementary Material: Supplementary Figures.pdf

Supplementary Material
Click here to download Supplementary Material: Supplementary Figures.pdf

\begin{abstract}
Click here to download Supplementary Material: Supplementary Figures.pdf
\end{abstract}

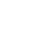

ff

(1)

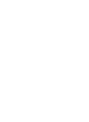

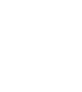

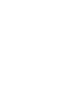

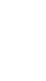

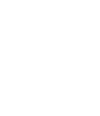

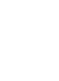

.

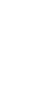
. (1)

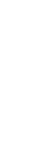

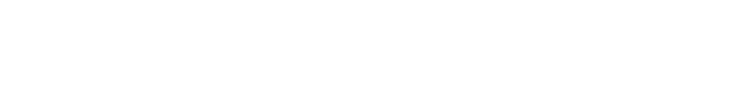
. 